\title{
Extensive circadian and light regulation of the transcriptome in the malaria mosquito Anopheles gambiae
}

\author{
Samuel SC Rund', James E Gentile ${ }^{2}$ and Giles E Duffield ${ }^{1 *}$
}

\begin{abstract}
Background: Mosquitoes exhibit $24 \mathrm{hr}$ rhythms in flight activity, feeding, reproduction and development. To better understand the molecular basis for these rhythms in the nocturnal malaria vector Anopheles gambiae, we have utilized microarray analysis on time-of-day specific collections of mosquitoes over $48 \mathrm{hr}$ to explore the coregulation of gene expression rhythms by the circadian clock and light, and compare these with the $24 \mathrm{hr}$ rhythmic gene expression in the diurnal Aedes aegypti dengue vector mosquito.
\end{abstract}

Results: In time courses from An. gambiae head and body collected under light:dark cycle (LD) and constant dark (DD) conditions, we applied three algorithms that detect sinusoidal patterns and an algorithm that detects spikes in expression. This revealed across four experimental conditions 393 probes newly scored as rhythmic. These genes correspond to functions such as metabolic detoxification, immunity and nutrient sensing. This includes glutathione S-transferase GSTE5, whose expression pattern and chromosomal location are shared with other genes, suggesting shared chromosomal regulation; and pulsatile expression of the gene encoding CYP6M2, a cytochrome P450 that metabolizes pyrethroid insecticides. We explored the interaction of light and the circadian clock and highlight the regulation of odorant binding proteins (OBPs), important components of the olfactory system. We reveal that OBPs have unique expression patterns as mosquitoes make the transition from LD to DD conditions. We compared rhythmic expression between An. gambiae and Ae. aegypti heads collected under LD conditions using a single cosine fitting algorithm, and report distinct similarities and differences in the temporal regulation of genes involved in tRNA priming, the vesicular-type ATPase, olfaction and vision between the two species.

Conclusions: These data build on our previous analyses of time-of-day specific regulation of the An. gambiae transcriptome to reveal additional rhythmic genes, an improved understanding of the co-regulation of rhythms in gene expression by the circadian clock and by light, and an understanding of the time-of-day specific regulation of some of these rhythmic processes in comparison with a different species of mosquito. Improved understanding of biological timing at the molecular level that underlies key physiological aspects of mosquito vectors may prove to be important to successful implementation of established and novel insect control methods.

\section{Background}

The mosquito An. gambiae is the primary African malaria vector, whilst $A e$ e aegypti is the primary vector of dengue fever and yellow fever. Mosquito physiology and behavior are under rhythmic control, organized in a time-of-day specific manner. Eukaryotic organisms possess a circadian ("about a day") clock, regulating daily

\footnotetext{
* Correspondence: duffield.2@nd.edu

'Department of Biological Sciences and Eck Institute for Global Health, Galvin Life Science Center, University of Notre Dame, Notre Dame IN 46556, USA Full list of author information is available at the end of the article
}

rhythms in biochemistry, physiology and behavior. It is cell autonomous, and at the molecular level is comprised of a series of transcriptional-translational feedback loops (TTFLs), whose completion takes approximately $24 \mathrm{hr}$ [1]. In An. gambiae daily behavioral rhythms are known to include dusk mating swarms, nocturnal flight activity, sugar feeding, blood feeding and oviposition. Late day larval-pupal ecdysis and late day/early night eclosion are also rhythmic [2-14].

Ae. aegypti behavioral rhythms have been described from populations collected or observed in the field from

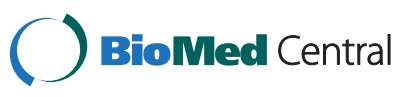


around the world as diurnal (often with increased activity during the first and last few hours of the daytime, i.e. crepuscular). These diel/circadian rhythms include flight activity, oviposition, host seeking, human landing/ biting and sugar feeding [14-27].

The role of specific An. gambiae clock genes in the lightinhibition of blood feeding behavior was revealed by DNA microarray analysis and RNAi-mediated gene silencing [10]. Studies of the mosquito canonical clock components include the cloning of the Ae. aegypti timeless gene (tim, AAEL006411) [28]; brain in situ hybridization of $A e$. aegypti cycle (cyc, AAEL002049) [29]; the expression profiling of clock genes in Ae. aegypti, An. gambiae, and Culex quinquefasciatus $[24,28,30]$; the functional analysis of the cytochrome proteins, CRY1 (AGAP001958) and CRY2 (AGAP004261) in An. gambiae [31,32]; and geographic and developmental variations in expression of timeless in the pitcher plant mosquito, Wyeomyia smithii [33].

Recently, we reported in Rund et al. genome-wide profiling of rhythmic gene expression in female mated but non-blood-fed An. gambiae heads and bodies under both LD (light:dark cycle, $11 \mathrm{hr}$ full light, $11 \mathrm{hr}$ darkness, and 1 hr dawn and dusk transitions) and DD (constant dark) conditions [30]. This work revealed genes involved in processes such as immune response, detoxification, transcription, oxidation/phosphorylation, translation, fatty acid metabolism, glycolysis/gluconeogenesis, olfaction, visual transduction and cuticle-related genes to be rhythmically expressed in An. gambiae. Under LD conditions, this included 1293 and 600 rhythmic genes with a period length of $20-28 \mathrm{hr}$ in the head and body, respectively, representing 9.7 and $4.5 \%$ of the An. gambiae gene set [30]. We studied heads and bodies separately because we expected enrichment (and thus increased detectability) of different genes in the different body segments; for example vision and antennal olfaction-related genes in the head, and genes in the body associated with gut, fat body, and skeletomuscular functions. Under DD conditions, we identified 891 rhythmic transcripts in the head and 476 in the body with an 18.5-26.5 hr period length [30]. A study of Ae. aegypti mosquitoes performed by Ptitsyn et al. [34], that profiled rhythmic gene expression analysis in the heads of female Ae. aegypti mosquito under LD conditions, also revealed transcriptional rhythms in gene expression across a wide variety of biological processes. Our analysis of An. gambiae rhythms utilized the COSOPT algorithm to mine expression data, whilst Ptitsyn et al., report results from the Fisher's g-test, autocorrelation and the Pt-test algorithm. The COSOPT cosine-wave fitting algorithm [35-38] is one of several, and arguably the method most used to mine gene expression data for genes rhythmically expressed with a sinusoidal expression pattern [36,37,39-43]. Other methods for identifying sinusoidal expression patterns include the recent JTK_CYCLE algorithm [44-46] and Fourier transform [47-49]. Investigations in maize, mice and artificially generated transcript profiles, for example, have demonstrated differing results in number and identity of genes scored as rhythmic depending on the algorithm used [39,44]. Additionally, there are non-sinusoidal yet still $24 \mathrm{hr}$ patterns of expression, such as pulsatile "spikes" which were noted in maize and Arabidopsis thaliana circadian transcriptional analysis using HAYSTACK $[39,50]$, which may be missed by algorithms searching specifically for sinusoidal expression patterns. We note male and female An. gambiae mosquitoes have an abrupt onset and short duration of elevated flight activity at dusk under both LD and DD conditions $[13,30]$, and therefore we hypothesized this could correspond with "spike" gene expression profiles.

Rhythmic genes exhibiting a $24 \mathrm{hr}$ period length are generated through the intersection of two processes: 1) The first is an endogenous circadian clock that persists under constant environmental light and temperature conditions (true "circadian" expression). The persistence of behavioral, physiological, and/or gene expression rhythms under constant conditions is thus indicative of an endogenous clock. 2) The second is a direct action of the environmental LD cycle on the organism that generates additional diel rhythms (rhythms observed under LD but not necessarily DD conditions) in gene expression and suppresses a proportion of rhythms generated by the endogenous circadian clock mechanism. This direct LD cycle mechanism has been described in Drosophila and our An. gambiae studies, yet is poorly understood at the molecular level. It presumably includes photoreception, including a contribution from the compound eyes $[30,37,48,51]$.

In this work, we reanalyze our original An. gambiae data using the more recently developed JTK_CYCLE algorithm, as well as perform a discrete Fourier transform (DFT) analysis. We use the consensus from these two methods along with our original COSOPT analysis to identify more genes as rhythmic with a high degree of confidence. We use a pattern matching algorithm novel to biological analyses to identify genes displaying clear pulsatile "spikes," since genes displaying this pattern may be missed by the other algorithms. Next, we further investigated the intersection between light-driven and endogenous clock-driven expression of rhythmic genes by looking at some unique patterns in gene expression that are present as mosquitoes make the transition from LD to DD conditions. We examine the presence of defined transcriptional regulation motifs in the $5^{\prime}$ upstream regions (presumed promoter regions) of those genes. Finally, we also reanalyze the Ae. aegypti gene expression data of Ptitsyn et al. using JTK_CYCLE and compare patterns in $~ 24 \mathrm{hr}$ rhythmic gene expression in the head under LD conditions between An. gambiae and Ae. aegypti across a variety of biological 
functional categories. This is interesting because both species of mosquitoes are vectors of disease, but may show different diel/circadian expression patterns owing to differences in temporal niche (An. gambiae is strictly night active and $A$ e. aegypti primarily day active), evolutionary lineage, and/or habitat [52,53]. Improving our understanding of the biology of these vectors (and recognizing the differences between them) is important in generating new methods of control at a time when there is emerging resistance of the mosquito to insecticide and resistance of the malaria parasite to drug treatment [54-56].

\section{Results and discussion}

Analysis of An. gambiae time course data with COSOPT, JTK_CYCLE and discrete Fourier transform reveals new rhythmic probes

Our original analysis [30] of the rhythmic nature of the mosquito transcriptome used very strict criteria to reduce the likelihood of false positives, at the expense of several obvious false negatives. In order to expand this analysis and identify previously unidentified rhythmic transcripts, we reexamined our microarray data to identify novel rhythmic expression patterns at high confidence using an approach of applying multiple algorithms to the same dataset $[34,39,47]$. We first reanalyzed our microarray data from An. gambiae [30], which was originally analyzed using the COSOPT algorithm, using DFT and the more recently developed JTK_CYCLE algorithm. All three of these algorithms search array data for sinusoidal rhythmic expression patterns, but variations in the methods leads to different results. In Additional file 1 we provide the number of probes we identified as rhythmic in each of our four experimental collection conditions (LD heads, DD heads, LD bodies and DD bodies) using various statistical cutoff thresholds. Different cutoff values have been used in various reported studies in an effort to balance the number of rhythmic genes reported against incidents of false positives. In our original COSOPT analysis we used a conservative cutoff of the multiple means corrected $\beta$ $(\mathrm{pMMC} \beta)$ of $\mathrm{p}<0.1$, in an attempt to minimize the occurrences of false-positives. However, in the current analysis we considered probability values as high as $\mathrm{p}<0.2[42,57]$.

In heads under LD conditions, when considering the least stringent cutoff values, COSOPT $(\mathrm{p}<0.2)$, JTK cycle $(\mathrm{q}<0.1)$ and DFT $(\mathrm{s}>0.3)$ each returned $\sim 2300$ probes determined to be rhythmic. The statistical cutoff values for COSOPT and JTK_CYCLE match the highest thresholds values utilized elsewhere, whilst the DFT value was chosen as it returned approximately the same number of probes $[42,44,57]$. When we considered the overlap of probes found rhythmic by using each of these three algorithms, 1658 probes were determined to be rhythmic by all three methods (Figure 1). Of these 1658 probes, 159 were not identified as rhythmic using the COSOPT criteria from our previous report [30]. New rhythmic probes were also identified in LD bodies, DD heads and DD bodies, where 148, 47 and 32 probes, respectively, were determined to be rhythmic that were not identified as such in our previous analysis (Additional file 2). Note that DFT analysis limits the number of probes that may be deemed rhythmic under DD conditions; see methods for more information. We believe that these newfound rhythmic genes can be called rhythmic with a high degree of confidence, since three separate algorithms identified them as such. Similar to our previous analysis [30] we found additional rhythmic genes in a range of functional groups dominated by metabolism, but also rich in detoxification, immunity, and cuticular function (see Additional file 3). From the LD head analysis, several of these newly found rhythmic probes reference genes of unknown function, or map to genomic regions not currently identified as genes.

Our reanalysis of microarray data using alternate expression-mining algorithms resulted in the identification

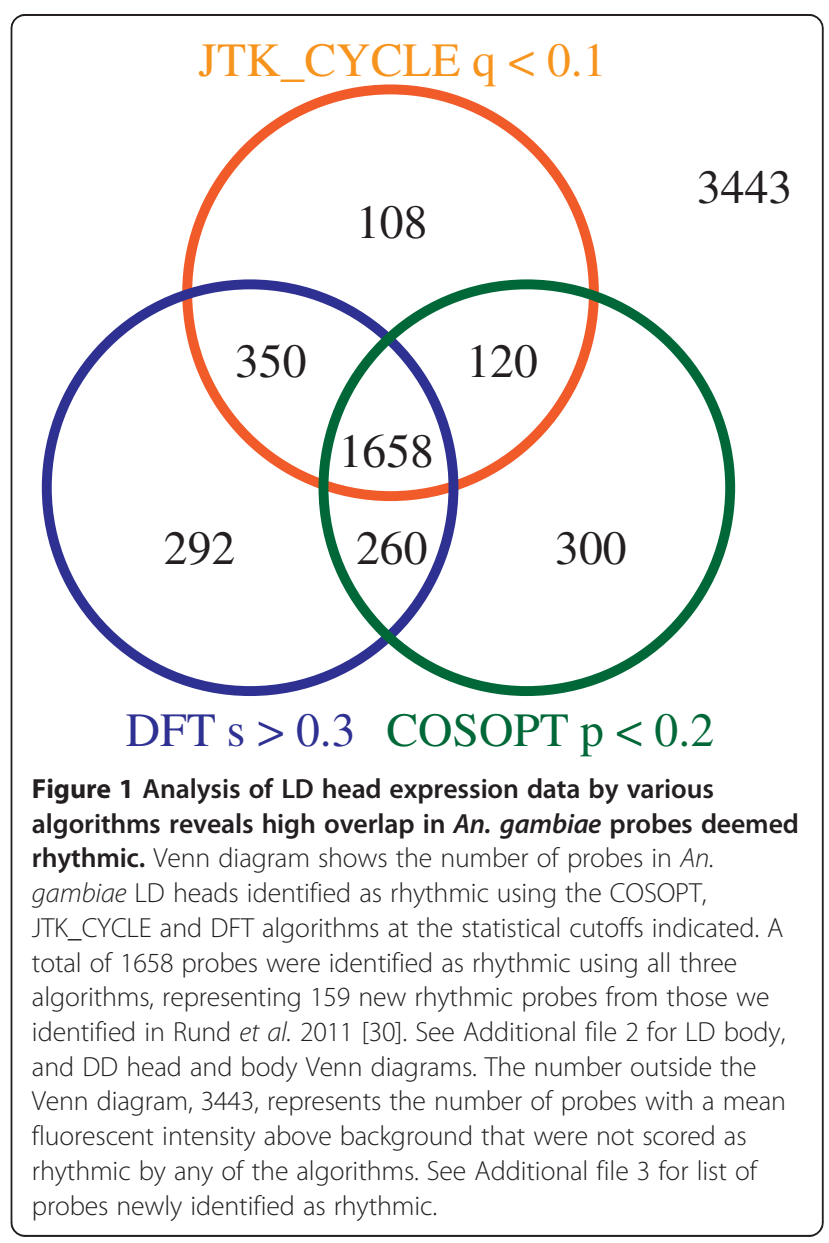


of more rhythmic genes that could underlie important rhythmic mosquito physiological processes - notably, detoxification, immunity and nutrient sensing genes. All time course expression profiles, including COSOPT and JTK_CYCLE outputs, can be viewed on our publically accessible database, Bioclock [58]. The discovery of more rhythmic genes adds more evidence in An. gambiae for rhythmic susceptibility to factors such as insecticide, infection and environmental challenges, as well as targets for manipulation to disrupt important rhythmic mosquito biological processes. Recent work in the closely related mosquito, Anopheles funestus, has shown that populations of these important malaria vectors are shifting their biting times in response to the utilization (and therefore selective pressure) of insecticide treated bednets [59]. Future investigations into this phenomenon should consider the current work presented here, as a shift in the expression of one or several of the genes we report as rhythmic might explain or underlie the reported shift in behavior.

\section{Detoxification genes newly identified as rhythmic}

Detoxification genes newly identified as rhythmic include the glutathione S-transferase (GST), GSTE5 (AGAP009192), which is noteworthy as it joins GSTE3 (AGAP009197) and GSTE2 (AGAP009194), two other GSTs on division 33B of polytene chromosome arm 3R [60] that we previously found rhythmically expressed in LD heads [30]. GSTE2 is a known resistance gene with a gene product that has been confirmed to metabolize DDT [60]. These three genes share nearly identical times of peak expression, potentially indicating a shared gene regulatory process. Chromosomal regions of rhythmic coregulation have also been noted in Drosophila [61]. In LD bodies we found five more rhythmically expressed annotated or predicted detoxification genes including cytochrome P450 6P4 (CYP6P4, AGAP002867) and GSTD11 (AGAP004378) (Additional file 3). All five of these detoxification genes we had previously identified as rhythmic in DD bodies, but not in LD bodies [30].

\section{Immunity and nutrient sensing/feeding genes newly identified as rhythmic}

Further, the list of genes newly found rhythmic under LD conditions includes components of An. gambiae immune gene families including the clip-domain serine protease new to our rhythmic list, CLIPD5 (AGAP002813, head), and CLIPE6 (AGAP011785), previously identified as rhythmic in LD heads and now in LD bodies; the class b scavenger receptor, agSCRB8 (AGAP004845), previously identified as rhythmic in the body but now head; and the serine protease inhibitor (serpin), SRPN5 (AGAP009221), previously identified as rhythmic in LD and DD heads and now in LD and DD bodies (Additional file 3).
Finally, our previous analysis revealed numerous genes that are involved in nutrient sensing and/or feeding behavior in various conditions/tissues including the takeout genes (TO1, AGAP004263; TO2 and/or TO3, AGAP012703/AGAP004262), adipokinetic hormone receptor (AKHR, synonymous with gonadotropin-releasing hormone receptor, GPRGNR1, AGAP002156), target of rapamycin (TOR, AGAP007873), neuropeptide F (NPF, AGAP004642), and the Anopheles homologues to Drosophila Lipid storage droplet-1 (LSD1, AGAP002890), SNF1A/ AMP-activated protein kinase (agAMPK, AGAP002686) and foraging (for, AGAP008863) [30]. In subsequent work, we revealed time-of-day dependent increases in flight behavior in An. gambiae and Ae. aegypti by pharmacological activation of the protein kinase G (PKG) encoded by the for gene [14]. This is of particular interest as dengue virus infection increases Ae. aegypti flight activity behavior [62] and PKG mediates a phosphorylation event involved in dengue virus replication [14].

We now find agAMPK (peak phase, ZT 4-ZT 6) and a predicted forkhead domain transcription factor (in Drosophila, foxo) (AGAP008606, peak phase ZT 9) additionally rhythmic in the body; new to the rhythmic list, the Anopheles homologue to Drosophila sugarbabe (sug, AGAP006736) was found rhythmic in the body and peaking at the end of the night phase (ZT 22-ZT 0) (Additional file 3). Drosophila sug encodes a predicted zinc finger protein that regulates insulin gene expression in neurosecretory cells [63], whilst Drosophila FOXO regulates the insulin receptor pathway [64].

\section{Using a pattern matching algorithm to search for pulsatile expression patterns}

The COSOPT, JTK_CYCLE and DFT algorithms all search for sinusoidal expression patterns. However, expression of genes that may have a $24 \mathrm{hr}$ rhythmic but non-sinusoidal pattern, and contribute to the rhythmic biology of the organism, may be overlooked by these three algorithms (i.e. pulsatile expression patterns). For example, daily onset of flight activity under LD and DD conditions is abrupt and highly elevated [13,30], and we hypothesized that there are phase-coincident pulses ("spikes") of gene expression associated with such transient behavior. We therefore utilized a pattern matching algorithm to search for expression patterns that were pulsatile, corresponding to spikes in expression with an interval of $24 \mathrm{hr}$. While we were unable to identify any genes with pulsatile expression under DD conditions (contrary to our hypothesis), we identified 11 genes in the LD heads and 5 in LD bodies with such a pattern (see Figure 2A). Some pulsatile genes were still found to be rhythmic by COSOPT independently, but two of the body genes, a homologue of Drosophila $N p c 2 d$ (AGAP002851) and a putative copper oxidase gene 


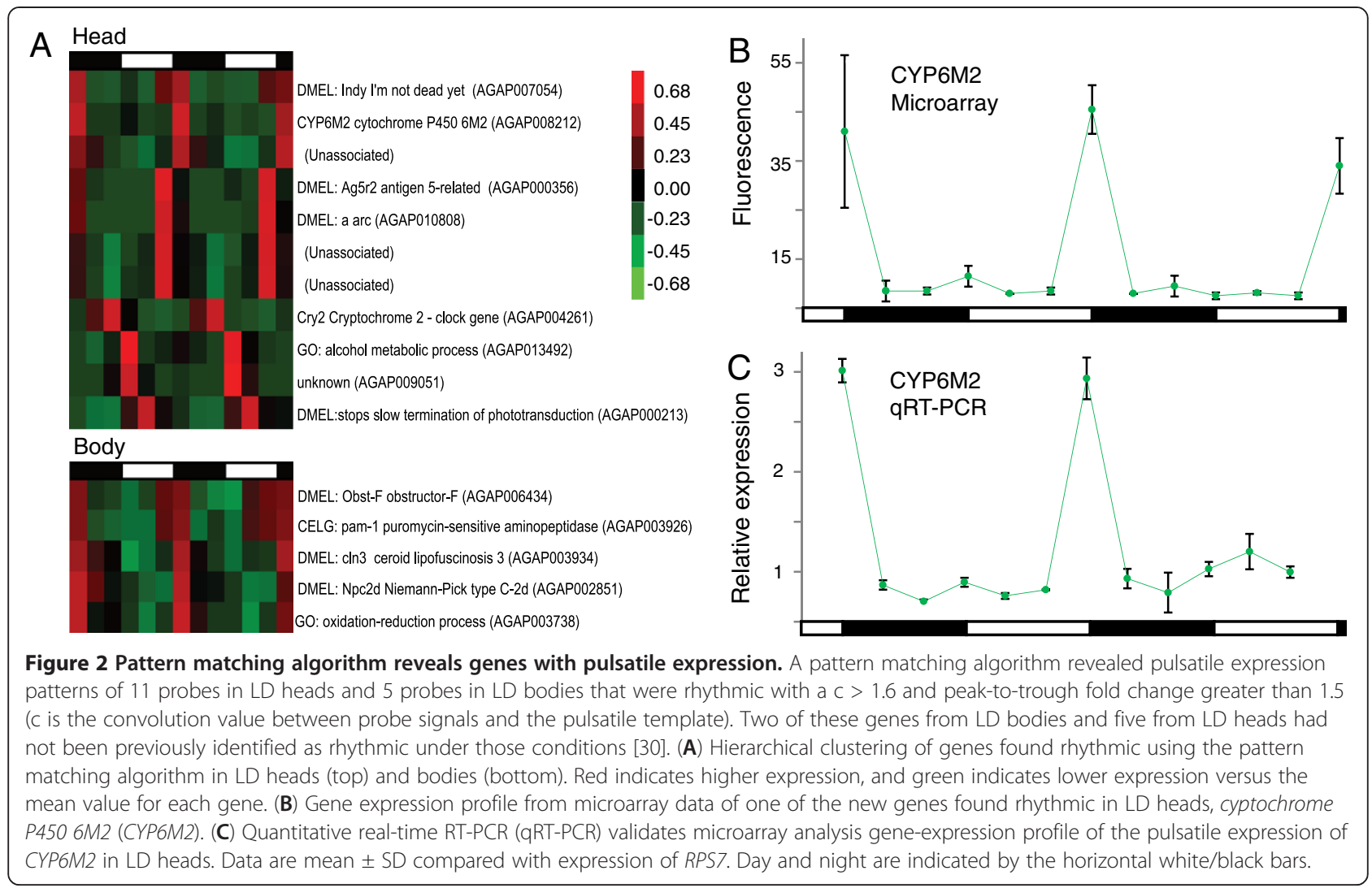

(AGAP003738), were not previously identified as rhythmic [30]. Similarly, in the head we found five genes with pulsatile expression patterns not found as rhythmic in our previous COSOPT analysis [30]. These are a homologue to Drosophila antigen 5-related (Ag5r2, AGAP000356); a homologue to the Drosophila transporter, I'm not dead yet (Indy, AGAP007054); a Drosophila homologue to $\operatorname{arc}(a$, AGAP010808); a gene predicted to play a role in alcohol metabolism, AGAP013492 (which was also detected as rhythmic in DD bodies); and a gene of unknown function (AGAP009051). Most interesting, however, was the pulsatile expression of CYP6M2 (AGAP008212) (Figure 2B), a cytochrome P450 monooxygenase that is known to be upregulated in insecticide resistant mosquitoes [65], and has a gene product demonstrated to metabolize pyrethroids [43]. CYP6M2 was previously identified as rhythmic in Anopheles bodies, but not heads [30]. In order to confirm our microarray expression data, we performed qRT-PCR on LD head samples and indeed, confirmed a pulsatile expression pattern of CYP6M2 (Figure 2C).

\section{Light regulation of the An. gambiae transcriptome}

A significant role of light in regulating gene expression and behavior in the mosquito is becoming apparent
$[10,30]$. In this section, we show that there are a large proportion of $A n$. gambiae genes that have expression at least partially driven (or suppressed) by the LD cycle. We show that the odorant binding proteins (OBPs) display three interesting patterns of gene expression as the mosquitoes transition from LD to DD conditions, and propose mechanisms that may underlie the control of gene expression in this subset of genes. For example, rhythmic gene expression can be driven by the LD cycle, the circadian clock, or the interaction of the two (Figure 3A) [30,37,48,51]. Changes in gene expression can be induced in An. gambiae through a short light pulse [10], while changes in host seeking or locomotor activity patterns in Ae. aegypti and D. melanogaster, respectively, can be altered through the modification of light regimes $[66,67]$. In this section we focus on the mechanism through which the LD cycle may influence rhythmic gene expression. Our previous study revealed more genes to be rhythmic (COSOPT, p < 0.1) under LD conditions than under DD, specifically 1293 versus 891 in heads and 600 versus 476 in bodies, although some genes were rhythmic only under DD conditions [30]. We hypothesized that the difference in the number of genes found rhythmic in the two conditions is primarily the result of a direct action by light in driving (or suppressing) rhythmic gene expression 


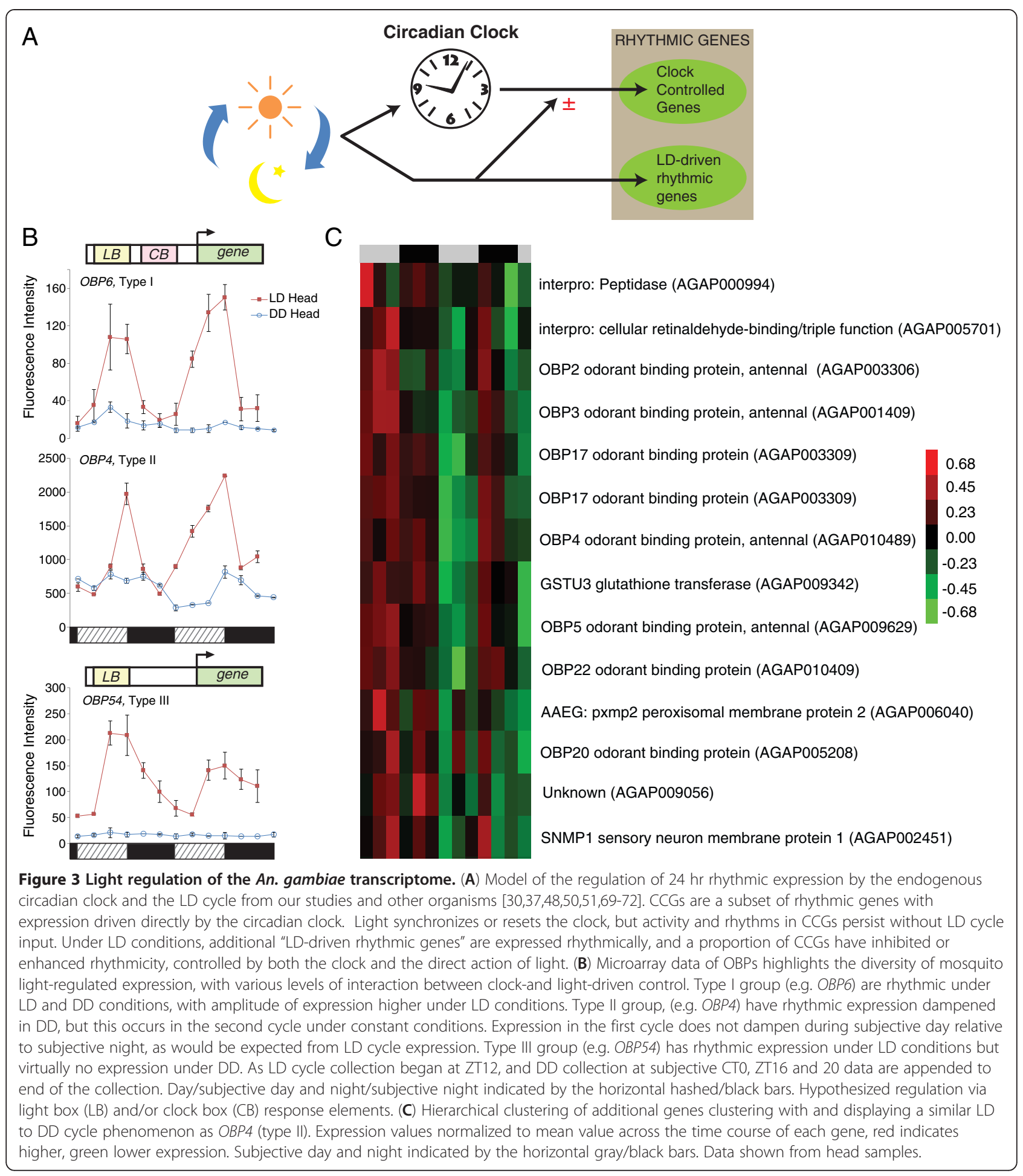

in the mosquito [30] (Figure 3A). At the regulatory level, we propose light box (LB, or light response element) and/or clock box ( $\mathrm{CB}$, or clock response element) promoters may drive the rhythmic expression of particular gene(s) [68] (see below for a discussion on this mechanism).

\section{Olfactory genes in particular highlight different potential} mechanisms of clock- and light-driven gene regulation An. gambiae olfactory genes, and in particular those encoding the OBPs, provided interesting examples of different mechanisms that could underlie rhythmic expression. 
OBPs are soluble proteins that facilitate the activation of olfactory receptors by transporting odor molecules through the antennal lymph to the receptors in the olfactory membrane [73-75]. Many of these OBP genes we previously found to be rhythmic in the head under LD conditions, peaking around dusk (ZT 12) but not under DD [30] (no additional OPBs were found rhythmic in the new expanded rhythmic list, above). Further inspection, however, revealed three interesting patterns in rhythmic expression exhibited by the olfactory genes as the mosquitoes transitioned from LD to DD conditions (i.e. differences in gene expression between a $24 \mathrm{hr}$ day in LD, the first $24 \mathrm{hr}$ day under DD conditions and the second $24 \mathrm{hr}$ day under DD conditions). We define these expression patterns as types I, II and III.

The type I group, OBP6 (AGAP003530; see Figure 3B), OBP7 (AGAP001556), OBP14 (AGAP002905) and OBP26 (AGAP012321), showed rhythmic expression under LD and DD conditions, but with dramatic reduction in expression under DD conditions versus LD conditions. In these genes, expression under DD conditions in the first cycle ( $24 \mathrm{hr}$ period) was similar to the second cycle (next $24 \mathrm{hr}$ period), with expression increasing during subjective day and falling during subjective night. These two observations suggest that expression of these genes is driven by the action of the circadian clock and the LD cycle through clock boxes and light boxes working in concert. The Clock Box $(\mathrm{CB})$ is a cis-acting site that is essential for rhythmicity, whereas the Light Box (LB) mediates most of the light-induced regulation [68].

The type II group contained OBP2 (AGAP003306), OBP3 (AGAP001409), OBP4 (AGAP010489; see Figure 3B), OBP5 (AGAP009629), OBP17 (AGAP003309) and OBP22 (AGAP010409). The expression levels of these genes is similar to the type I group with its dramatically reduced expression in DD versus LD; however, in the LD to DD cycle transition, expression of these type II genes does not dampen during subjective day (circadian time, CT 0 - CT 12) under the first cycle in $D D$ relative to subsequent cycles (Figure 3B). From this, we can deduce that these genes are all presumably under control of both a $\mathrm{CB}$ and a LB that act in concert to drive rhythmic expression at higher amplitude than by the clock alone. Under LD conditions, the clock and light work together to drive robust, high amplitude rhythms in expression. As the mosquitoes transition from LD to DD, there is an initial transition cycle in DD where there is still dependency on inputs from the LD cycle and thus the genes display irregular expression patterns. Finally, in subsequent cycles in DD, rhythmic expression is driven entirely by the clock. To see if other genes might have similar expression patterns, we performed hierarchical cluster analysis of DD head expression on the subset of probes identified as rhythmic under LD conditions (in the expanded list, above) to search for additional genes with similar expression patterns as these type II OBPs. We found 13 genes (14 probes) with similar expression including those for the olfaction gene, sensory neuron membrane protein 1 (SNMP1, AGAP002451) [76] and the detoxification gene, glutathione transferase U3 (GSTU3, AGAP009342) [77] (Figure 3C). All of the clustered genes showed a lower level of expression in DD in the same manner as the type II group of OBPs. This pattern of expression under DD conditions suggests that these 13 genes are under control of both a $\mathrm{CB}$ and a LB. Indeed, 5 of these genes, the olfaction genes OBP7, OBP22, OBP26 and SNMP1, and the immunity gene, galectin 3 (GALE3, AGAP004934), have previously been shown to be downregulated in the head following acute light treatment presented during late night [10,78].

The type III group of genes, OBP51 (AGAP006077), OBP29 (AGAP012331), OBP47 (AGAP007287), OBP54 (AGAP006080, see Figure 3B) and OBP57 (AGAP011368), are rhythmic only under LD conditions. Under DD conditions we see these genes are expressed at or below the nadir level of expression observed under LD conditions. We predict that rhythmic expression of these genes would be driven exclusively by a LB, with no contribution from the circadian clock.

For OBP6 (type I) and OBP3 (type II), we confirmed using qRT-PCR a reduction in expression in DD as compared to LD conditions. In mosquitoes studied concurrently under different lighting conditions, expression under DD conditions at CT 12 was found to be at $23 \pm 5 \%$ and $27 \pm 34 \%$ (mean \pm SD) of expression levels under LD conditions at ZT 12 (Additional file 4A). Furthermore, when we look at the mean expression level across $44 \mathrm{hrs}$ of genes rhythmic under LD conditions (in the expanded list, above), we find that while most probes showed nearly identical expression between LD and DD heads, significant variation between LD and DD expression levels does occur in a smaller subset of genes. The difference in bodies was more pronounced, where $47 \%$ of rhythmic body genes show >2-fold differential expression in DD compared with LD (Additional file 4B).

These data reveal a complex interaction between clock-derived signals and photic signals that act on the regulation of OBPs in particular, but also on other genes such as GSTU3 and SCRB1. In fact, specific genes found in all three groups have been previously reported to show reductions in their expression following a light pulse presented during the late night phase of the LD cycle. These include OBP26 (type I), OBP22 (type II) and OBP47 (type III) [10]. Moreover, these gene expression changes are correlated with suppressed feeding behavior, and in fact, manipulation using RNAi knockdown of OBP4 (type II group) results in altered blood-feeding behavior [10]. Clearly, the current findings are particularly interesting as it highlights the potential for manipulating 
the mosquito olfactory system, and thus perhaps behavior, through timed light exposure. Indeed, OBPs 47, 3, 7, 17, 4 and 22 that we describe here are likely involved in host seeking as they are enriched at least 2-fold higher in female than male antennae [73].

\section{The role of light regulation and the molecular circadian clock in rhythm generation}

To explore further the effect of light on the regulation of rhythmicity, we also examined in the head the amplitude of the canonical clock components PER (AGAP001856), TIM (AGAP008288), CRY2 (AGAP004261), CYC (AGA P005655) and PDP1 (AGAP006376), identified as rhythmically expressed in An. gambiae (COSOPT, p < 0.1; JTK_CYCLE, q <0.05) [30]. For PER, TIM and CRY2, we find a consistently smaller peak-to-trough amplitude in the DD compared to LD conditions, a consistent reduction in the JTK_CYCLE algorithm determination of amplitude [44], and a sequential reduction in amplitude between the first and second cycle in DD that is not apparent between cycles in LD conditions (Additional file 5). For $C Y C$ there was variability between probes in the condition effect, and for PDP1 rhythm amplitude between conditions was lower. However, no reduction between the first and second cycle in DD was detected. This dampening of the key elements of the transcriptional translational feedback loop (TTFL) of the circadian clock in DD has been observed in Drosophila [79-81].

To understand the potential mechanism through which light independently regulates these rhythms in An. gambiae, we must turn to genetic model organisms such as Drosophila. Genetic deletion of the clock has revealed that some LD rhythms are independent of the circadian pacemaker [48]. Amplitude of output processes does however appear to be coupled to the TTFL of the clock, but evidence for this dependency is mixed $[82,83]$. For example, the rhythms in Drosophila clock proteins PER and TIM, clock controlled gene (CCG) expression and locomotor behavior, do persist even when their corresponding per or tim gene expression is artificially held constant [84].

It is plausible that the small level of dampening in the rhythms of elements of the TTFL observed in $A n$. gambiae within the first two cycles in DD could contribute to changes in CCG expression. However, it is unlikely that it would be the primary cause for the dramatic loss or reduction in rhythmicity observed for many CCGs, such as the OBPs.

At least in the rhythms observed in the head, it is likely that the compound and simple eyes contribute to the mechanism of light regulation. In Drosophila mutant for the intracellular photoreceptor dCRY (CRY1 in the mosquito), flies are still responsive to light and their LD cycle-driven rhythms persist [48]. However, flies with a mutant phospholipase $\mathrm{C}$ component of phototransduction, NORPA (no receptor potential A), have a loss of light regulated rhythms [48]. In the mammalian clock, discrete signaling by light and by the clock is apparent in the regulation of the immediate early genes and/or clock genes $c$-fos, mPer 1 and mPer2 [85]. Light in this case results in transient gene expression that is associated with resetting of the clock, and light acts indirectly via the $\mathrm{Ca}^{2+} / \mathrm{cAMP}$ response element (CRE). In contrast, the clock components act upon the E box element(s) in the promoter regions of these genes.

At least based on precepts primarily from the Drosophila system, we would propose a model for An. gambiae to explain our results that consists of: i) separate clock response element(s) or 'clock box' (CB) and light response element(s) or 'light box' (LB) within the promoters of rhythmic genes; and/or ii) the action of light signaling impinging upon pathways upstream of the CB but downstream of the TTFL. This model is not unreasonable given the complexity of light/circadian regulation being uncovered in genetic model species from several taxonomic groups $[48,50,68,82,83,86,87]$.

\section{Clock- and light-regulated response element gene promoter search}

In an attempt to identify potential circadian clock- and light- response elements we next searched for promoter elements identified in Drosophila as contributing to rhythmic gene expression. Specifically, we searched the $5 \mathrm{~kb} 5$ region upstream of the transcription start sites in type I OBPs, type II OBPs and the other genes with similar expression patterns (see Figure $3 \mathrm{C}$ ), and type III OBPs, for E boxes (from the very generic CANNTG to the canonical CACGTG sequence), W boxes, CREs, Per repeat (PERR) elements, Tim-E-box-like repeat (TER) elements and PDP1 binding sites (PDP1s) [49,88-95]. We find that all 22 genes show examples of at least two different consensus sequences within their upstream region (Additional file 6). We find the occurrence of one or more TER sequences in the upstream regions of all genes except for OBP14 and OBP57 (which we note both have upstream regions of $<1.8 \mathrm{~kb}$ ). W boxes and CREs also appear well represented across all groups with at least one occurrence in 12 and 9 upstream gene regions, respectively. We note no PERRs or PDP1s were found in any type III OBPs. These promoter sequences are considered to be definitive clock regulatory elements $[91,94,95]$. PERR elements were found only in type II genes, with 3 examples of gene upstream regions that have at least one occurrence. PDP1s appeared in 2 of 4 type I upstream regions and 6 of 13 type II regions. Surprisingly, the presence of consensus sequences implicated in clock-regulation including $\mathrm{W}$ boxes, TER 
elements and canonical E boxes, were found extensively in the promoter regions of type III genes. Finally, we find that 9 genes from across all types have a least one occurrence of CREs in the upstream promoter regions, which is not surprising as all type I, II and III genes appear to be at least partially regulated by the direct action of the LD cycle. CREs in mammals are critical to transducing light information to the clock [85], and is plausible that CREs may also contribute to light-regulated expression of the OBPs and other genes in the mosquito.

\section{Comparisons between rhythmic gene expression in Ae. aegypti and An. gambiae}

Recently, rhythmic expression profiling of the Ae. aegypti mosquito was performed in a similar manner to our An. gambiae transcriptional profiling [34]. With the publication of these data, we were able to undertake a detailed comparison of rhythmic gene expression between the two species and describe our results in this final section.

Both species of mosquitoes are vectors of disease, but may show different diel/circadian expression patterns owing to differences in temporal niche, evolutionary lineage [52], and/or habitat [53]. An. gambiae is strictly nocturnal in its patterns of flight activity, sugar and host seeking, blood feeding, mating, and ovipostion behavior [2-4,7-12,14,30,96-100], whilst Ae. aegypti is diurnal, primarily active during the mid-late afternoon (i.e. ZT 6-12, where ZT 12 is defined as lights off) [14-16,20-25, $27,101,102]$. If we consider flight activity behavior for example, $A n$. gambiae is active throughout the night and rests exclusively during the day, as well as shows a transient elevation of activity at the end of dusk/early night phase, coincident with swarming behavior. Ae. aegypti is most active during the latter half of the day/ light phase, and tends to show peaks in activity at dawn/early morning and especially so at the end of the day/dusk (i.e. crepuscular); Ae. aegypti shows little or no activity during the night. Coincident with flight activity, similar temporal patterns have been shown in the field and laboratory for biting behavior: with An. gambiae biting occurring during the night, and Ae. aegypti during the morning and late afternoon.

A better understanding of the differences and similarities, and thus potentially different physiological or behavioral responses, in rhythmic gene expression between these two species may prove important in the design and implementation of future control strategies. As an example, we recently demonstrated that when Ae. aegypti and An. gambiae females were injected with a pharmacological protein kinase G (PKG) activator, 8-pCPT-cGMP (Guanosine-3'-5' -cyclic Monophosphate, 8-(4-Chlorophenylthio)), both species showed several days of increased flight/wing beat activity, but only at the times of the $24 \mathrm{hr}$ day of their normal flight activity profile when they would normally be active [14].

In order to make as similar as possible comparison of rhythmic gene expression between the two species, from experiments of slightly different design, we reanalyzed both datasets using JTK_CYCLE with identical criteria, a stringent $\mathrm{q}<0.05$ probability cutoff and a period length of 20-28 hr. Interestingly, when we look at the distribution of peak phases (the number of genes which have their peak in expression at any particular time of the day) we find that $A n$. gambiae have genes peaking in expression at all times of the $24 \mathrm{hr}$ day, but an enrichment in the number of genes peaking at the dawn and dusk transitions. Ae. aegypti, however, has a low percentage of genes with rhythmic expression profiles peaking during ZT 11-17 (first two-thirds of the night phase) (Figure 4A). Interestingly, this is coincident with the nightly Ae. aegypti rest period.

Table 1 presents the number of genes from the various biological categories that we found rhythmic in $A n$. gambiae (a total of 1400 rhythmic An. gambiae genes), the number of those genes where an Ae. aegypti homologue is identified in VectorBase (a total of 1202 An. gambiae genes had an Ae. aegypti homologue), and the number of those 1202 Ae. aegypti genes that were rhythmic themselves (a total of 539 genes). See Additional file 7 for details of the 539 common genes. Overall, we confirmed that the Ae. aegypti transcriptome is highly rhythmic (4475 genes were identified as rhythmic using JTK_CYCLE), and many genes rhythmic in $A n$. gambiae have homologues that are also rhythmic in Ae. aegypti.

We then looked at individual categories of genes to compare their expression patterns between Aedes and Anopheles, and report here on some of the categories of rhythmic genes that we found that had interesting differences or similarities in expression patterns between the two species. We hypothesize how differences in diel expression between the two species could be explained by differences in known circadian biology between the two species as has been suggested in other studies between animals in different temporal niches [24,105-108]. However, we acknowledge that as we are only comparing two species, this present analysis can only conclusively show the presence of a difference between the two species, and not the reason for such differences.

\section{Temporal similarities and differences in V-ATPase gene expression between An. gambiae and Ae. aegypti}

The multi-subunit vesicular-type ATPase (V-ATPase) that utilizes ATP to actively transport $\mathrm{H}^{+}$, has been detected in Ae. aegypti in the osmoregulatory tissues, including stomach, malpighian tubules, anterior hindgut and rectum [109]; in An. funestus salivary glands [110]; and in the antennal sensilla of the saturniid moth Antheraea pernyi 


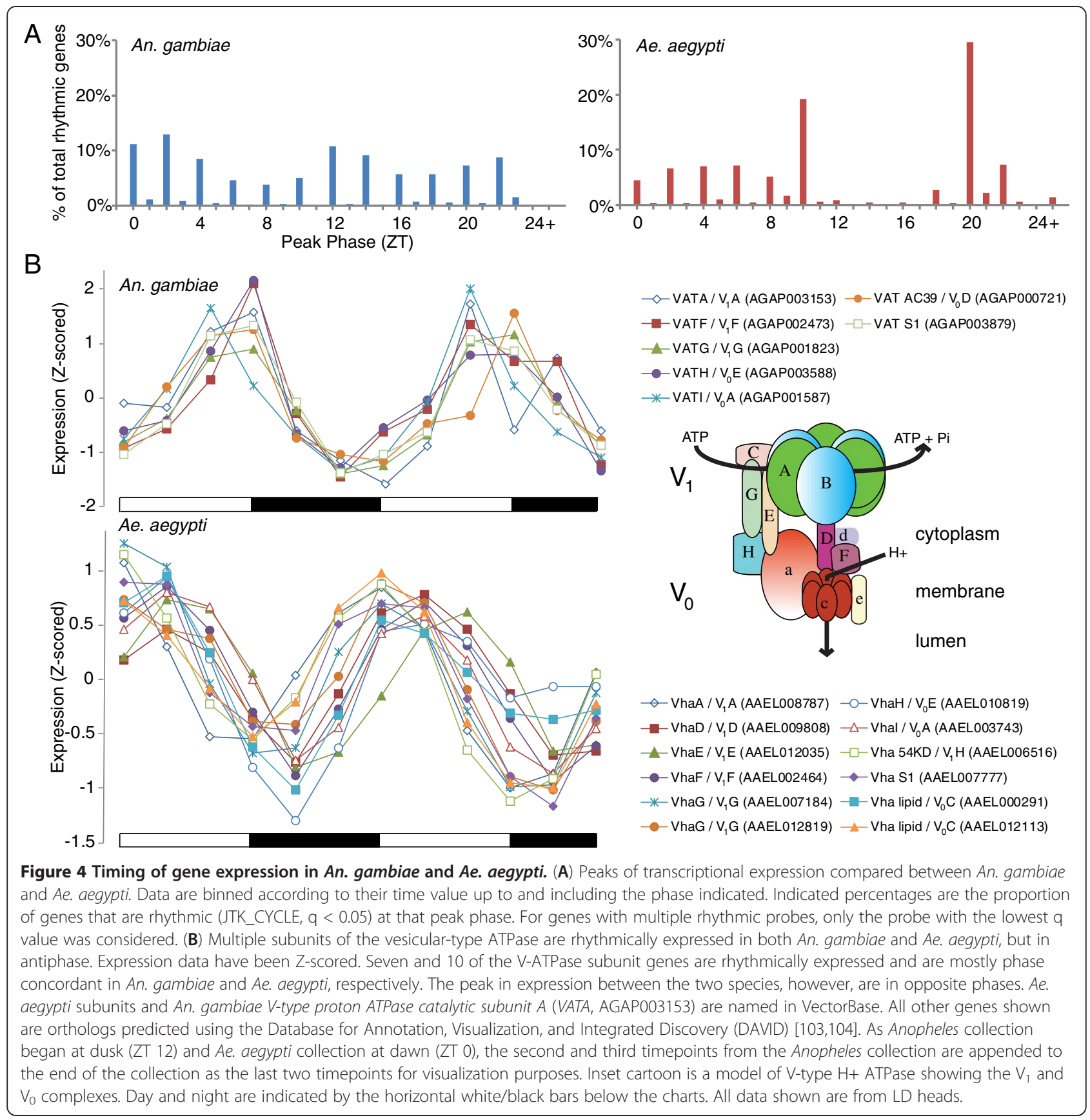

[111]. Importantly, there is evidence for a role of VATPase in Plasmodium infection in Aedes and Anopheles, and dengue and Japanese encephalitis infections in Aedes [112-114]. We have previously commented on the relevance of coordinated rhythms in V-ATPase subunit expression specifically in the bodies of An. gambiae, and its potential relationship to Plasmodium infection of the mosquito mid-gut $[30,114]$. V-ATPase is also thought to play a crucial role in the function of synaptic vesicles, and indeed Drosophila mutant for the $\mathrm{V}_{0}$ subunit a1 have impaired neurotransmitter release [115-117]. This mutation also impacts the endolysosomal degradation mechanism in Drosophila eye photoreceptors [118], and the Drosophila B-subunit V-ATPase is rhythmic at the protein level in the eye [119]. In the head under LD conditions, and using DAVID $[103,104]$ to identify orthologs, we found that 7 genes encoding 7 of the 12 subunits in Anopheles to be rhythmic and in phase, with all peaking in the late day/ dusk. In Aedes, 12 of the subunit genes (that represent 10 of the 12 subunits) are rhythmic and also expressed at the same phase, but the peak in expression is in opposite phase to An. gambiae, occurring around dawn (Figure 4B). 
Table 1 Comparing rhythmic An. gambiae genes to Ae. aegypti rhythmic genes

\begin{tabular}{lccc}
\hline Category & $\begin{array}{c}\text { Rhythmic } \\
\text { Anopheles } \\
\text { genes }\end{array}$ & $\begin{array}{c}\text { Aedes } \\
\text { homologues }\end{array}$ & $\begin{array}{c}\text { Rhythmic } \\
\text { Aedes } \\
\text { homologues }\end{array}$ \\
\hline Chromatin Modification & 19 & 17 & 8 \\
Detoxification & 38 & 35 & 18 \\
Immunity & 47 & 40 & 25 \\
Metabolism & 222 & 207 & 99 \\
Neuronal/Behavior & 43 & 39 & 16 \\
Olfaction & 29 & 28 & 8 \\
Other & 106 & 103 & 39 \\
Protein Folding/Modification & 43 & 39 & 21 \\
Proteolysis & 47 & 42 & 16 \\
Redox & 54 & 51 & 24 \\
Signal Transduction & 79 & 72 & 29 \\
Structural & 47 & 43 & 18 \\
Transcription & 68 & 63 & 30 \\
Translation & 49 & 46 & 15 \\
Transport & 123 & 46 & 49 \\
Unknown & 373 & 319 & 120 \\
Vision & 13 & 12 & 4 \\
Totals: & $\mathbf{1 4 0 0}$ & $\mathbf{1 2 0 2}$ & $\mathbf{5 3 9}$ \\
\hline The Ist of Angmber & 49 & & 24 \\
\hline
\end{tabular}

The list of An. gambiae genes found rhythmic in An. gambiae heads under LD conditions from reanalyzed Rund et al. 2011 data [30] with a JTK_CYCLE q $<0.05$ cutoff are compared to Ae. aegypti heads under LD conditions from Ptitsyn et al. 2011 [34] data also reanalyzed with JTK_CYCLE q < 0.05 cutoff. For each biological functional category, the number of genes found rhythmic in An. gambiae, the number of those genes where a homologue was identified in Ae. aegypti, and finally the number of those homologues that were found rhythmic in Ae. aegypti is provided.

As V-ATPase subunit gene expression is rhythmic, our analysis highlights the possibility that susceptibility by Aedes to dengue and Japanese encephalitis viruses may vary by time of day. Furthermore, if the rhythms are in similar phase in Aedes bodies as they are in their heads, it is likely that these mosquitoes up-regulate their V-ATPase at times when significant osmotic changes induced by a blood or sugar meal at differing if not opposite times of the day from Anopheles may occur, concordant with known differences in behavioral rhythms. Finally, as $\mathrm{V}$-ATPase plays an important role in synaptic activity, it is possible that neuronal activity is modulated in a time-of -day manner in the two species, yet in opposite phases, again concordant with the differing times of behavioral activity in each mosquito.

Temporal similarities in vision gene expression between An. gambiae and Ae. aegypti

We next looked at genes involved in the visual transduction pathway, using the Drosophila visual signaling pathway [120-122] as a model to identify mosquito orthologs, and identify genes rhythmic in both Anopheles and Aedes (Figure 5). The eye specific ninaA/cyclophilin-r (AGAP009991/AAEL009421) encoding an eye-specific cyclophilin which is involved in rhodopsin transport from the endoplasmic reticulum [123], peaks in expression in both Anopheles and Aedes in the early morning phase. Particularly interesting is the inaD signaling complex. The inaD protein organizes components of the phototransduction cascade into a signaling complex that contains, among other components, the kinase/myosin hybrid, ninaC (AGAP009730/AAEL000596). Expression of ninaC is rhythmic in both species, peaking at mid- to late night. In Anopheles, but not Aedes, expression of inaD (AGAP002145/AAEL008705) itself, as well as another gene encoding a component of the signaling complex, retinophilin (rtp, AGAP003547/AAEL000457) is rhythmic [30]. In Aedes however, the major light-gated ion channel, trp (AAEL005437), is rhythmic, peaking in expression in the early morning. Expression of trp (AGAP000348) was not detected on our An. gambiae microarray. Finally, in both Anopheles and Aedes, stops (AGAP000213/ AAEL005443) is rhythmically expressed, peaking at mid-day. The PLC $\beta$ regulator, STOPS, is critical for maintaining protein, but not mRNA, levels of NORPA [124] suggesting conserved rhythmic control of visual signal transduction could be tightly regulated by NORPA through rhythmic expression of norpA as well as through STOPS. The rhythmic gene expression of visual transduction proteins in Anopheles and Aedes might contribute to a conserved time-of-day specific gating mechanism for tuning sensitivity to photic activation of the mosquito visual system irrespective of temporal niche (i.e. nocturnal versus diurnal) to match the daily changes in light levels. This is consistent with electrophysiological studies in numerous other insect species [125]. Organisms that fail to adjust their sensitivity to light in a time-of-day manner will have visual systems too insensitive during the night and overly sensitive during the day [125].

\section{Temporal differences in aminoacyl-tRNA synthetases and olfaction gene expression between An. gambiae and Ae. aegypti}

We next compared gene expression between the putative aminoacyl-tRNA synthetases that "prime" amino acids to tRNA, and the olfaction genes $O B P \mathrm{~S}$ and odorant receptor coreceptor (orco), between the two mosquito species. In $A n$. gambiae, we found significant rhythmic co-regulation with 11 rhythmic aminoacyltRNA synthetases $(q<0.05)$ that all peak approximately in phase toward the later part of the night in LD heads (Figure 6). In $A n$. aegypti, we find 15 aminoacyl-tRNA synthetases are rhythmic (Figure 6), and that there is an enrichment in genes peaking in expression towards the middle of the day (antiphasic to Anopheles) but with 


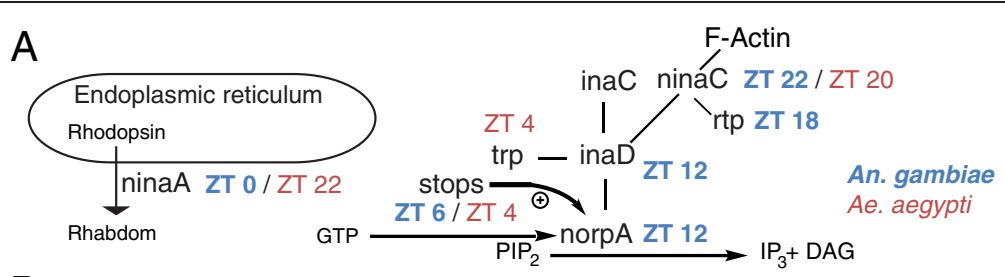

B
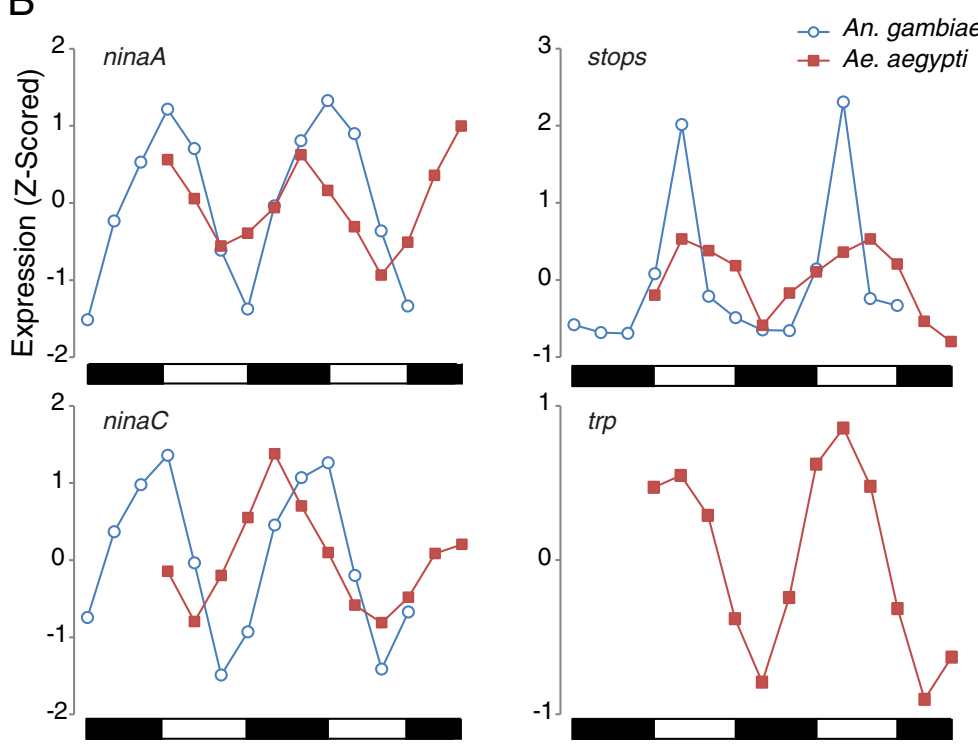

Figure 5 Multiple components of the visual transduction cascade are rhythmically expressed and in similar phases in both An. gambiae and Ae. aegypti. (A) Mosquito homologues to genes in the Drosophila phototransduction cascade signaling complex were identified as rhythmic in LD heads using the JTK_CYCLE algorithm $(q<0.05)$. Peak phase in expression is indicated next to gene names as Zeitgeber time (ZT) with An. gambiae genes in bold blue and Ae. aegypti genes in red. (B) Transcription profiles of genes rhythmic in both species as well as Ae. aegypti transient receptor potential (trp). An. gambiae trp expression was not detected above background levels. Expression values are Z-scored. Day and night are indicated by the horizontal white/black bars below the chart. The shift in the presentation of the beginning and end of expression profiles reflects differences in experimental design between Anopheles and Aedes collections. Mosquito visual gene identities and functions are based on homology to Drosophila and are presumed similar in Anopheles and Aedes mosquitoes [120,121,126]. For the full set of An. gambiae vision genes found rhythmic, see Rund et al. 2011 [30]. All data shown are from LD heads.

several exceptions (genes peaking in expression at other times of the day). Observed rhythms in aminoacyl-tRNA synthetases would suggest that mosquitoes have increased protein synthesis activity during their behavioral inactive periods. This could correspond with the rebuilding of cellular products while the mosquito rests, as well as anticipation of large amounts of protein synthesis involved in egg development that follow a blood meal. Our results indicate there may be rhythmic control at the translational level which produces, enhances or modifies $24 \mathrm{hr}$ rhythms downstream of gene expression. As the peak in expression of the aminoacyl-tRNA synthetases is different between the nocturnal $A n$. gambiae and diurnal Ae. aegypti, we hypothesize that in both species, expression of aminoacyl-tRNA synthetases is upregulated prior to the mosquitoes' inactive phase in preparation for increases in protein synthesis while the mosquito is in a rest state.
A similar pattern of co-regulation existed in the olfactory genes that we examined. In An. gambiae there appears to be very tight regulation among the 17 rhythmic $O B P$ s $(\mathrm{q}<0.05)$, with a majority peaking in expression around dusk. However, the 15 rhythmic OBPs in Ae. aegypti peak in expression at various times of the day, instead of clustering around a particular phase of the LD cycle. This finding may be related to when there may be temporal segregation of behaviors requiring the detection of discrete odors. Finally, we compared the expression of the gene encoding the master olfactory heterodimer required for all odorant receptor transduction, odorant receptor coreceptor (orco) between the two species (AGAP002560/AAEL005776) [128]. Note in $A n$. gambiae, orco is also known as odorant receptor 7 (OR7). We find that orco ( $\mathrm{q}=0.06)$ peaks in An. gambiae at ZT 10 , which is immediately prior to dusk (ZT 12) and the onset of nocturnal behavioral activities involving olfaction, i.e. host seeking, blood feeding, nectar feeding and oviposition 


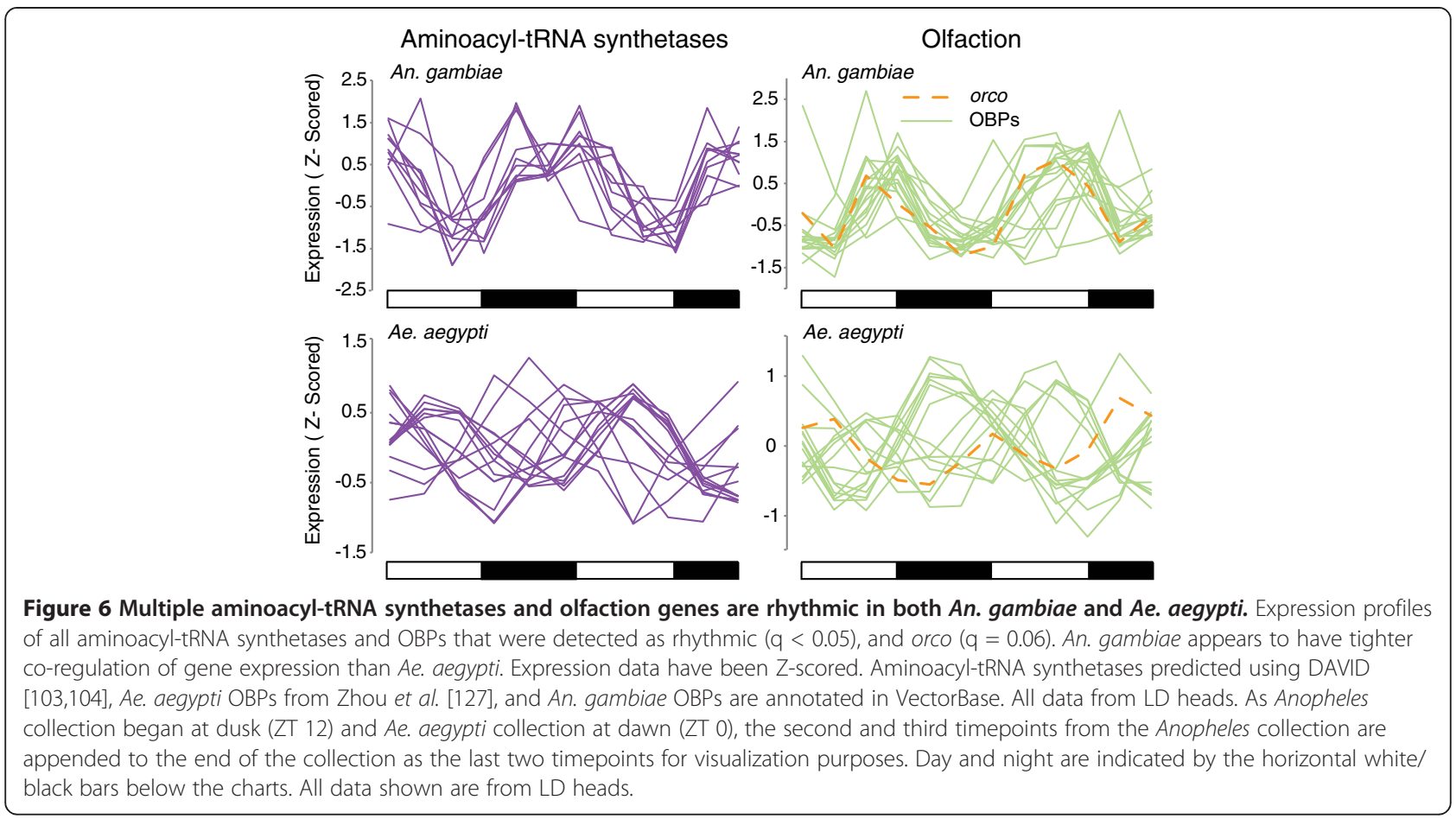

[3-12]. However, orco peaks in the morning at ZT 3 in Ae. aegypti, which may be consistent with this species being most active during the day time $[15,16,21,25,101]$.

\section{Conclusions}

Mosquitoes exhibit $24 \mathrm{hr}$ time-of-day specific rhythms in flight activity, feeding and reproductive behaviors and developmental processes. To understand the molecular basis for these rhythms in An. gambiae, we have utilized microarray analysis on $48 \mathrm{hr}$ time courses collected from female heads and bodies. Recent studies have highlighted a broad diversity of $24 \mathrm{hr}$ rhythmic gene expression in nocturnal An. gambiae and diurnal Ae. aegypti mosquitoes, although no previous comparison of rhythmic genome-wide expression between the two temporally segregated species has been made. In An. gambiae, many genes are rhythmic only in an environmental LD cycle suggesting direct regulation of gene expression by light, whilst others are rhythmic under DD conditions, revealing regulation by the endogenous circadian clock. In time courses from An. gambiae head and body under LD and DD conditions, we applied three algorithms that detect sinusoidal patterns and an algorithm that detects spikes in expression. This revealed across four experimental conditions 393 probes newly scored as rhythmic. These genes correspond to functions such as metabolic detoxification, immunity and nutrient sensing. Included are GSTE5, whose expression pattern and chromosomal location are shared other with other GSTs, and suggests shared chromosomal regulation; the pulsatile expression of CYP6M2, a cytochrome P450 that metabolizes pyrethroid insecticides; and the Anopheles homologue to Drosophila sugarbabe, a regulator of insulin synthesis. Time course expression profiles and cosine wave-fitting algorithm data for all probes can be viewed on our publically accessible database, Bioclock [58]. In total, between the present study and our previous we have revealed under LD conditions, 1424 and 726 rhythmic genes with a period length of $20-28 \mathrm{hr}$ in the head and body, respectively; and under DD conditions, 928 rhythmic genes in the head and 510 in the body with an $18.5-26 \mathrm{hr}$ period length.

We explored the interaction of light and the circadian clock and highlight the regulation of OBPs that are important components of the olfactory system. We reveal that $O B P$ s have unique expression patterns as mosquitoes make the transition from LD to DD conditions, and propose a model for the three distinct patterns of expression that we observe. Finally, we compared rhythmic expression between time courses of An. gambiae and Ae. aegypti heads collected under LD conditions using a single cosine fitting algorithm, and report distinct similarities and differences in the temporal regulation of genes involved in key processes such as protein synthesis (specifically tRNA priming), the V-ATPase and in the sensory modalities of olfaction and vision. We propose that the similarities and differences shared between the two species may in part reflect their distinct temporal niches, although they also have differences in habitat and evolutionary lineages which likewise could be underlying the differences we report $[52,53]$. 
These data build on our previous analyses of the timeof-day specific regulation of the An. gambiae transcriptome. Improved understanding of the molecular basis for circadian- and light-regulated rhythms that underlie key physiological aspects of mosquito vectors may prove to be important to successful implementation of established and novel vector control methods. Rhythmic changes in genes associated with susceptibilities to immune and insecticidal challenges, sensory physiology and feeding behavior may provide opportunities for new control strategies, including gene manipulation by generation of transgenic mosquitoes [129,130]. Other important implications of such extensive rhythmic regulation includes the efficacy of sterile insect technique/pathogen-resistant strains, where differences in diel timing of mating between reared and wild populations would limit their success [129-132]. Moreover, the use of insecticide impregnated bed nets may be acting as a selective pressure that is modifying the age/genetic composition of the population and the time when nocturnal anopheline vectors initiate host-seeking behavior such that it occurs at a different time of the night $[59,133]$. These considerations illustrate the need for a better understanding of the circadian biology of these disease vector species.

\section{Methods}

\section{Microarray gene expression data}

An. gambiae microarray data collection and analysis were originally reported in Rund et al. 2011 [30]. In that study, female mated, but not blood-fed, Pimperena S molecular form mosquitoes were collected every $4 \mathrm{hr}$ over $48 \mathrm{hr}$ under either LD or DD conditions, heads separated from bodies, RNA extracted, and RNA expression levels determined using the Affymetrix platform (Plasmodium/ Anopheles Affymetrix GeneChips, Affymetrix 900511). Expression data is deposited in GEO Express (accession no. GSE22585), VectorBase Expression Data BioMart [134] and are graphically available and easily searchable at our website, Bioclock [58].

Ae. aegypti microarray data collection and analysis was originally reported in Ptitsyn et al. 2011 [34]. In this study, female mosquitoes (as a mixed population from 11 separate $F_{5}$ colonies originally derived from wild caught populations from Chiapas, Mexico) were collected in duplicate every $4 \mathrm{hr}$ over $24 \mathrm{hr}$ under LD conditions, RNA extracted from heads, and RNA expression levels determined using Agilent microarrays described in $\mathrm{Xi}$ et al. [135]. Normalized gene expression data as $44 \mathrm{hr}$ profiles was available as supplementary material to their publication [34], and reanalyzed and presented here as a derivative work in accordance with the terms of the Creative Commons 2.0 license and the BioMedical Central open access charter.

\section{Application of COSOPT, JTK_CYCLE and discrete Fourier transform algorithms for generating a consensus rhythmic gene list}

An. gambiae microarray data comprised two replicate samples. Only An. gambiae expression profiles where mean fluorescent intensity > 20 (which would exclude 99\% of Plasmodium falciparum probes contained on the microarray) in both replicates were considered. No fluorescent intensity cutoff was applied to Ae. aegypti probes.

Software implementations of the COSOPT [30,35-38, 42,43] and JTK_CYCLE [44-46] algorithms were used as previously described. Briefly, COSOPT measures the goodness-of-fit between experimental data and a series of cosine curves with varying phases and (user defined) period lengths. Multiple means corrected $\beta$ (pMMC $\beta$ ) values are determined by scrambling experimental data and re-fitting it to cosine curves to determine probability that the observed data matches a cosine curve by chance alone. JTK_CYCLE is a nonparametric statistical algorithm designed to identify and characterize cycling variables in large data sets. It applies the Jonckheere-Terpstra-Kendall (JT) test and Kendall's tau (rank correlation), finding the optimal combination of period and phase that minimizes the p-value of Kendall tau correction between the experimental time series and each tested cyclical ordering, this being derived from cosine curves. JTK_CYCLE generates period length and phase estimates, as well as corrects for multiple comparisons post hoc (the p-value for a given probe is converted to a more stringent BenjaminiHochberg q-value, which takes into consideration the possible false positive rate across all probes.) A measure of rhythm amplitude is also determined and reflects the 1 -cycle median sign-adjusted deviation from the median in relation to the optimal cosine pattern [for a perfect cosine wave, this is amplitude/sqrt(2), where amplitude is defined as the median absolute deviation from the median].

For COSOPT and JTK_CYCLE, only probes with an average computed period length of between $18.5-26.5 \mathrm{hr}$ for constant condition experiments or 20-28 hr for LD experiments in both replicate time courses were considered. The multiple means corrected $\beta$ (pMMC $\beta$ ) value, $p$, was used as the described COSOPT cutoff value. In this paper, described $\mathrm{p}$ value cutoffs are the averages of the two COSOPT-generated $\mathrm{p}$ values from each of the replicate time courses.

For JTK _CYCLE, the Benjamini-Hochberg q-value was used as the described JTK_CYCLE cutoff value. JTK_CYCLE accounts for replicate samples, thus only one $q$ value is generated.

For discrete Fourier transform (DFT), the relative amplitude of the $24 \mathrm{hr}$ period frequency component was calculated with the discrete Fourier transform [136]. 
First the time series data was transformed by

$$
X=|\operatorname{DFT}(x)|
$$

where $\mathrm{x}$ is the time series signal and $\mathrm{X}$ is a vector of the sinusoidal amplitudes. To mitigate the effects of the mean fluorescent intensity, $\mathrm{X}[0]$ was set to zero. Note that since the sampling rate is $4 \mathrm{hr}$ with a window of $48 \mathrm{hr}$, X has seven tuples and each value defines the amplitude of an $\mathrm{N} / 48 \mathrm{hr}$ embedded frequency where $\mathrm{N}$ is the index. Thus, as period lengths deviate farther from $24 \mathrm{hr}$, they are less likely to be discovered by this method. This becomes particularly apparent under DD conditions.

The relative amplitude of the $24 \mathrm{hr}$ period $(1 / 24 \mathrm{hr}$ frequency) component characterized the presence of that sinusoid in the data. This was calculated by

$$
s=X[2] /|X|
$$

ensuring that the value would range between zero and one. For any described s value cutoff, the average of the $s$ values returned from the two replicate time courses is considered.

\section{Pattern matching to search for pulsatile expression patterns}

Pulsatile patterns were discovered by convolving a template with the expression signals [137]. The template, which corresponds to spikes in expression, $24 \mathrm{hr}$ apart, was defined mathematically as

$$
T=\left[\begin{array}{llllllll}
1.0 & -0.4 & -0.4 & -0.4 & -0.4 & -0.4 & 1.0
\end{array}\right] .
$$

These values were chosen such that convolution with unity (constitutive, non-cyclic expression) is 0 and the peak samples are weighted more than the valleys. Prior to convolution, the signals were gamut normalized then reduced by the mean value of the signal. Convolution yielded a $\mathrm{c}$ value for each of the 13 time points; the maximum $\mathrm{c}$ value was used to represent the maximum pulsatile expression for each given expression pattern across the 13 time points. Expression profiles were considered pulsatile where $\mathrm{c}>1.6$ and where peak-to -trough fold change $>1.5$ in both replicates. The $\mathrm{c}$ value cutoff was determined through manual inspection as the threshold at which no apparent false-positives were detected. Note $\mathrm{c}$ has a magnitude and a sign. Highmagnitude, positive values reflect a good match to the template whereas small magnitude values reflect a poor match to the template.

\section{Gene annotation}

Where possible, we used the An. gambiae identifications from our previous report [30]. For genes not previously annotated, we used the same naming conventions. Briefly, genes were annotated primarily from information stored at VectorBase, often using the closest homologue from Ae. aegypti (AAEG:), Cx. quinquefasciatus (QQUI:), D. melanogaster (DMEL:) or Caenorhabditis elegans (CELG:) (in that order), but also using published literature and the Database for Annotation, Visualization and Integrated Discovery (DAVID) to match putative An. gambiae genes to enzymatic pathways $[103,104,134]$. Where no An. gambiae or orthologous gene name was available, InterProScan [138] was used to annotate genes; a representative InterPro or the associated Gene Ontology (GO) term may be provided. Ae. aegypti gene names were identified in a similar manner. Ae. aegypti OBPs were identified from Zhou et al. 2008 [127]. Gene annotations correspond with the July 3, 2012 VectorBase release. Genes that have been previously annotated by others in An. gambiae, but not in VectorBase, appear in the text with an 'ag' prefix.

\section{Hierarchical cluster analysis}

Hierarchical cluster analysis was performed using Cluster 3.0 and visualized using Java TreeView $[139,140]$. Data were $\log _{2}$ transformed, mean centered and normalized across the time course for each gene and clustered (centroid linkage). For An. gambiae, only probes that had a mean fluorescence intensity across all 13 timepoints $>20$ were analyzed.

\section{Real-time quantitative RT-PCR (qRT-PCR) analysis}

Total RNA was treated with DNaseI (Qiagen, Valencia, $\mathrm{CA}$ ) and used for cDNA synthesis using a High Capacity cDNA reverse transcriptase kit (Applied Biosystems, Foster City, CA) primed with random hexamers. PCR thermocycling and qRT-PCR were performed as previously described [35] using SYBR green reagents with an Applied Biosystems 7500 Fast Real-Time PCR System and quantification based on the generation of standard curves or the delta-delta CT method. Dissociation curves to test for primer dimers were generated using dissociation curve software (Applied Biosystems). Normalization of genes was calculated relative to ribosomal protein S7 (RPS7). Real-time quantitative RT-PCR Primer sequences $\left(5^{\prime} \rightarrow 3^{\prime}\right)$ : RPS7 (AGAP010592) F: CATTCTGCCCAAACCGATG, R: AACGCGGTCTCTT CTGCTTG, from Dana et al. 2005 [141]; CYP6M2 (AGAP008212) F: GTATGATGCAGGCCCGTATAG R: GCCATAATGAAACTCTCCTTCG from Müller et al. 2007 [142]; OBP3 (AGAP001409) F: GATTCGTGCT GGAGCTCGAG, R: GTAAAAAGTAGTGCACCGGGT CC; OBP6 (AGAP003530) F: CATGCTTAATGGATC TAACACAAAC, R: GCGACTTCACAGCGATCC from Biessmann et al. 2005 [143].

\section{Promoter search analysis}

A search for defined consensus sequences [49,88-94] was performed at the University of Notre Dame Genomics 
and Bioinformatics Core Facility using a custom written Perl script on the upstream regions of type I OBPs, type II OBPs and the other genes found clustering with those OBPs (see Figure 3) and type III OBPs. In general, we utilized the promoter element search criteria of ClaridgeChang et al. [49]. The upstream region of each gene of interest (up to $5 \mathrm{~kb}$ unless there was overlap with the predicted coding region of another gene) was downloaded from VectorBase [134]. See Additional file 6 for specific consensus sequences and search criteria.

\section{Comparison of genes rhythmic in An. gambiae versus Ae. aegypti}

In order to make as similar as possible comparison of rhythmic gene expression between the two species, from experiments of slightly different design, we reanalyzed both datasets using JTK_CYCLE with a stringent $\mathrm{q}<0.05$ probability cutoff of genes with a $20-28 \mathrm{hr}$ period. Using the list of gene homology maintained at VectorBase, homologues to all rhythmic An. gambiae genes were identified in Ae. aegypti. Homologues were then compared against the rhythmic Ae. aegypti gene list and matches noted. For both An. gambiae and Ae. aegypti the probe with the lowest q value was considered. The Ae. aegypti homologues considered were the homologues listed in VectorBase with the highest percent identity that were rhythmic $(\mathrm{q}<0.05)$.

\section{Additional files}

Additional file 1: Rhythmic An. gambiae probes, by statistical test cutoff value. Only probes with a mean fluorescent intensity $>20$ across the time course were analyzed. Probes indicated as rhythmic using COSOPT or DFT were found rhythmic in both of the two replicate runs. In JTK_CYCLE and COSOPT, only probes where period length under LD conditions was between $20 \mathrm{hr}$ to $28 \mathrm{hr}$ or in DD conditions between 18.5 $\mathrm{hr}-26.5 \mathrm{hr}$ are reported. Note DFT performed on $24 \mathrm{hr}$ signal for all runs, see methods for more details.

Additional file 2: Analysis of expression data by various algorithms reveals overlap in An. gambiae probes deemed rhythmic. Venn

diagrams show the number of probes in LD bodies and DD heads and bodies identified as rhythmic using the JTK CYCLE, DFT and COSOPT algorithms at the statistical cutoffs indicated. In LD bodies, a total of 808 probes were identified as rhythmic using all three algorithms,

representing 148 new rhythmic probes from those identified previously [30]. In DD heads, a total of 517 probes were found rhythmic using all three conditions (47 new probes). In DD bodies, a total of 332 probes were identified as rhythmic using all three algorithms (32 new probes). Note DFT analysis limits the number of probes that may be deemed rhythmic under DD conditions; see methods for more information. See Figure 1 for LD head Venn diagram. See Additional file 3 for list of probes newly identified as rhythmic. The numbers outside the Venn diagrams represent the number of probes with a mean fluorescent intensity above background that were not scored as rhythmic by any of the algorithms.

Additional file 3: An. gambiae probes found rhythmic by COSOPT, JTK_CYCLE and DFT but not in the original COSOPT analysis. List of probe identities for LD heads, DD heads, LD bodies and DD bodies found rhythmic with $\mathrm{pMMC} \beta<0.2$ (COSOPT), $\mathrm{q}<0.1$ (JTK_CYCLE), and $s$ $>0.3$ (DFT), but that were not found rhythmic using the original COSOPT statistical cutoff of $\mathrm{pMMC} \beta<0.1$ [30]. Only probes where the mean fluorescent intensity was $>20$ across all timepoints were considered. Annotation, probe ID, COSOPT pMMC $\beta$ and peak phase (ZT), JTK_CYCLE $\mathrm{q}$ and peak phase (ZT) and DFT $s$ values are provided. Probes that do not map to current genes are marked as "unassociated." Probes that map to more than one gene are marked with an asterisk.

Additional file 4: An. gambiae gene expression changes in LD versus DD conditions. (A) qRT-PCR confirmation of reduction in expression in OBP3 and OBP6 under DD versus LD conditions. Values are mean \pm SD of gene expression as a percentage normalized to the LD value of $100 \%$. Female mated, non-blood fed mosquitoes, 5-7 day post emergence from mosquitoes reared concurrently under different lighting conditions were collected under LD conditions or DD conditions (from mosquitoes placed in darkness for $24 \mathrm{hr}$ ) at ZT/CT 12. (B) Average gene expression changes between LD and DD conditions as measured by microarray analysis across $44 \mathrm{hr}$. The average expression level averaged across all 12 time points was analyzed in both LD and DD, and the fold change difference in expression level between LD and DD determined. Most probes showed similar expression levels between LD and DD. However, significant variation occured in a subset of genes. This was especially pronounced in bodies, where $47 \%$ of the rhythmic genes had $>2$ fold difference in expression levels between LD and DD conditions.

Additional file 5: Amplitude measures for An. gambiae clock genes expressed in the head under LD and DD conditions. Amplitudes calculated as peak divided by nadir normalized fluorescence values and where peak-to-nadir occurred with an interval of 8-16 hr. The JTK_CYCLE amplitude value reflects the 1-cycle median sign-adjusted deviation from the median in relation to the optimal cosine pattern.

Additional file 6: Promoter sequence search of light- and circadiandriven gene expression. Specific promoter search criteria and the results of searching for defined response elements [49,88-95] in the $5 \mathrm{~kb}$ 5' region upstream of the transcription start site of type I OBPs, type II OBPs and the other genes found clustering with those OBPs (see Figure 3), and type III OBPs. The table provides the gene name, VectorBase ID and the number and identity of consensus sequences found in the $1 \mathrm{~kb}$ and $5 \mathrm{~kb}$ upstream region of the genes. For some genes, the full $5 \mathrm{~kb}$ region was not available, as it would overlap with the predicted coding region of another gene. In such cases, only the region that did not overlap was considered; the number of base pairs considered is provided in the "Upstream region (bp)" column.

Additional file 7: Rhythmic genes in heads under LD conditions that are common to both An. gambiae and Ae. aegypti. 539 genes were identified as rhythmic $(q<0.05)$ in both An. gambiae and $A e$. aegypti. For each pair of homologous rhythmic genes, an An. gambiae annotation, JTK_CYCLE phases and q values, probe IDs and gene IDs are provided. For both An. gambiae and Ae. aegypti the probe with the lowest q value is provided. The Ae. aegypti homologues to An. gambiae that are provided in the table are those listed in VectorBase with the highest percent identity, that were also found rhythmic.

\section{Abbreviations}

CB: Clock box; CCG: Clock controlled gene; DD: Constant dark; CRE: $\mathrm{Ca}^{2+} / \mathrm{CAMP}$ response element; DFT: Discrete Fourier transform; GST: Glutathione S-transferase; LB: Light box; LD: Light:dark cycle; OBP: odorant binding protein; TTFL: Transcriptional - translational feedback loop; ZT: Zeitgeber time.

\section{Competing interests}

The authors declare no competing interests.

\section{Authors' contributions}

SSCR performed Anopheles and Aedes gene expression analysis, hierarchical cluster analysis, qRT-PCR and drafted the manuscript. JEG implemented the pattern matching algorithm, discrete Fourier transform and compared Anopheles and Aedes expression. GED conceived of the study and participated in its design, coordination and analysis and co-wrote the manuscript. All authors read and approved the final manuscript. 


\section{Acknowledgements}

We thank J. Hogenesch and M. Hughes for provision of and assistance with the COSOPT and JTK_CYCLE algorithms, G. Dimopoulos for provision of the Ae. aegypti array annotation, P. Zhou for assistance with qRT-PCR analysis, M. Allee for assistance with data processing techniques, S. Lee for assistance with manuscript preparation, R. Rund for review of the manuscript, and F. Collins for insightful discussions. We are grateful to the reviewers' suggestions that have improved the quality and readability of the manuscript. Funding was provided by the Genomics, Disease Ecology and Global Health Strategic Research Initiative and Eck Institute for Global Health, University of Notre Dame (pilot grants to GED and fellowship to SSCR).

\section{Author details}

'Department of Biological Sciences and Eck Institute for Global Health, Galvin Life Science Center, University of Notre Dame, Notre Dame IN 46556, USA. ${ }^{2}$ Department of Computer Science and Engineering, Fitzpatrick Hall, University of Notre Dame, Notre Dame IN 46556, USA.

\section{Received: 20 November 2012 Accepted: 14 March 2013}

Published: 3 April 2013

\section{References}

1. Dunlap JC, Loros JJ, Decoursey PJ: Chronobiology: Biological timekeeping. Sunderland Mass: Sinauer Associates; 2004

2. Charlwood JD, et al: The swarming and mating behaviour of Anopheles gambiae s.s. (Diptera: Culicidae) from São Tomé Island. J Vector Ecol 2002, 27:178-183.

3. Gary RE Jr, Foster WA: Diel timing and frequency of sugar feeding in the mosquito Anopheles gambiae, depending on sex, gonotrophic state and resource availability. Med Vet Entomol 2006, 20:308-316.

4. Jones MDR, Gubbins SJ: Changes in circadian flight activity of mosquito Anopheles gambiae in relation to insemination, feeding and oviposition. Physiol Entomol 1978, 3:213-220.

5. Reiter $P$, Jones MDR: Eclosion timing mechanism in the mosquito Anopheles gambiae. J Entomol Ser A 1976, 50:161-168.

6. Jones MDR, Reiter P: Entrainment of pupation and adult activity rhythms during development in mosquito Anopheles gambiae. Nature 1975, 254:242-244.

7. Jones MD: Delayed effect of light on the mosquito "clock". Nature 1973, 245:384-385.

8. Jones MDR, Cubbin CM, Marsh D: The circadian rhythm of flight activity of the mosquito Anopheles gambiae: The light-response rhythm. J Exp Biol 1972, 57:337-346.

9. Jones MDR, Hill M, Hope AM: The circadian flight activity of the mosquito Anopheles gambiae: Phase setting by the light regime. J Exp Biol 1967, 47:503-511.

10. Das S, Dimopoulos G: Molecular analysis of photic inhibition of bloodfeeding in Anopheles gambiae. BMC Physiol 2008, 8:23.

11. Fritz ML, et al: Ovipositional periodicity of caged Anopheles gambiae individuals. J Circadian Rhythms 2008, 6:2.

12. Sumba LA, et al: Daily oviposition patterns of the African malaria mosquito Anopheles gambiae Giles (Diptera: Culicidae) on different types of aqueous substrates. J Circadian Rhythms 2004, 2:6.

13. Rund SSC, Lee SJ, Bush BR, Duffield GE: Strain- and sex-specific differences in daily flight activity and the circadian clock of Anopheles gambiae mosquitoes. J Insect Physiol 2012, 58:1609-1619.

14. Keating JA, Bhattacharya D, Rund SSC, Hoover S, Dasgupta R, Lee SJ, Duffield GE, Striker R: Mosquito protein kinase G phosphorylates flavivirus NS5 and alters flight behavior in Aedes aegypti and Anopheles gambiae. Vector Borne Zoonotic Dis 2013, 13. in press.

15. Corbet PS, Smith SM: Diel periodicities of landing of nulliparous and parous Aedes aegypti (L.) at Dar es Salaam, Tanzania (Diptera, Culicidae). Bull Entomol Res 1974, 64:111-121.

16. Jones MDR: The programming of circadian flight-activity in relation to mating and the gonotrophic cycle in the mosquito, Aedes aegypti. Physiol Entomol 1981, 6:307-313.

17. McClelland GAH: Field observations on periodicity and site preference in oviposition by Aedes aegypti (L.) and related mosquitoes (Diptera: Culicidae) in Kenya. Proc R Entomol Soc Lond Ser A Gen Entomol 1968, 43:147-154
18. Tuchinda $\mathrm{P}$, Kitaoka M, Ogata $\mathrm{T}$, Kurihara $\mathrm{T}$ : On the diurnal rhythmus of biting behavior of Aëdes aegypti in relation to the age and to the hemorrhagic fever in Bangkok, 1964. Japan J Trop Med 1969, 10:1-6.

19. Harrington LC, Ponlawat A, Edman JD, Scott TW, Vermeylen F: Influence of container size, location, and time of day on oviposition patterns of the dengue vector, Aedes aegypti, in Thailand. Vector Borne Zoonotic Dis 2008, 8:415-424.

20. Kawada $\mathrm{H}$, Takagi M: Photoelectric sensing device for recording mosquito host-seeking behavior in the laboratory. J Med Entomol 2004, 41:873-881.

21. Yee WL, Foster WA: Diel sugar-feeding and host-seeking rhythms in mosquitoes (Diptera: Culicidae) under laboratory conditions. J Med Entomol 1992, 29:784-791.

22. Lardeux F, et al: Integrated control of peridomestic larval habitats of Aedes and Culex mosquitoes (Diptera: Culicidae) in atoll villages of French Polynesia. J Med Entomol 2002, 39:493-498.

23. Canyon DV, Hii JLK, Muller R: Effect of diet on biting, oviposition, and survival of Aedes aegypti (Diptera: Culicidae). J Med Entomol 1999, 36:301-308.

24. Gentile C, Rivas GBS, Meireles-Filho ACA, Lima JBP, Peixoto AA: Circadian expression of clock genes in two mosquito disease vectors: cry2 is different. J Biol Rhythms 2009, 24:444-451.

25. Chadee DD: The diel oviposition periodicity of Aedes aegypti (L.) (Diptera: Culicidae) in Trinidad, West Indies: Effects of forced egg retention. Bull Entomol Res 2010, 100:599-603.

26. Naucke TJ, et al: Field evaluation of the efficacy of proprietary repellent formulations with IR3535 and picaridin against Aedes aegypti. Parasitol Res 2007, 101:169-177.

27. Wong J, Astete H, Morrison AC, Scott TW: Sampling considerations for designing Aedes aegypti (Diptera:Culicidae) oviposition studies in Iquitos, Peru: Substrate preference, diurnal periodicity, and gonotrophic cycle length. J Med Entomol 2011, 48:45-52.

28. Gentile $C$, et al: Cloning and daily expression of the timeless gene in Aedes aegypti (Diptera: Culicidae). Insect Biochem Mol Biol 2006, 36:878-884.

29. Chahad-Ehlers S, Gentile C, Lima JBP, Peixoto AA, Bruno RV: Analysis of cycle gene expression in Aedes aegypti brains by in situ hybridization. PLoS One 2013, 8:e52559.

30. Rund SSC, Hou TY, Ward SM, Collins FH, Duffield GE: Genome-wide profiling of diel and circadian gene expression in the malaria vector Anopheles gambiae. Proc Natl Acad Sci USA 2011, 108:E421-E430.

31. Yuan Q, Metterville D, Briscoe AD, Reppert SM: Insect cryptochromes: Gene duplication and loss define diverse ways to construct insect circadian clocks. Mol Biol Evol 2007, 24:948-955.

32. Zhu H, Yuan Q, Froy O, Casselman A, Reppert SM: The two CRYs of the butterfly. Curr Biol 2005, 15:R953-R954.

33. Mathias D, Jacky L, Bradshaw WE, Holzapfel CM: Geographic and developmental variation in expression of the circadian rhythm gene, timeless, in the pitcherplant mosquito, Wyeomyia smithii. J Insect Physiol 2005, 51:661-667.

34. Ptitsyn $A$, et al: Rhythms and synchronization patterns in gene expression in the Aedes aegypti mosquito. BMC Genomics 2011, 12:153.

35. Duffield GE, et al: Circadian programs of transcriptional activation, signaling, and protein turnover revealed by microarray analysis of mammalian cells. Curr Biol 2002, 12:551-557.

36. Panda S, et al: Coordinated transcription of key pathways in the mouse by the circadian clock. Cell 2002, 109:307-320.

37. Ceriani MF, et al: Genome-wide expression analysis in Drosophila reveals genes controlling circadian behavior. J Neurosci 2002, 22:9305-9319.

38. Straume M: DNA microarray time series analysis: Automated statistical assessment of circadian rhythms in gene expression patterning. Methods Enzymol 2004, 383:149-166.

39. Khan S, Rowe SC, Harmon FG: Coordination of the maize transcriptome by a conserved circadian clock. BMC Plant Biol 2010, 10:126.

40. Hughes ME, et al: Harmonics of circadian gene transcription in mammals. PLoS Genet 2009, 5:e1000442.

41. Covington MF, Maloof JN, Straume M, Kay SA, Harmer SL: Global transcriptome analysis reveals circadian regulation of key pathways in plant growth and development. Genome Biol 2008, 9:R130.

42. Sato TK, et al: A functional genomics strategy reveals Rora as a component of the mammalian circadian clock. Neuron 2004, 43:527-537.

43. Stevenson BJ, et al: Cytochrome P450 6M2 from the malaria vector Anopheles gambiae metabolizes pyrethroids: Sequential metabolism of deltamethrin revealed. Insect Biochem Mol Biol 2011, 41:492-502. 
44. Hughes ME, Hogenesch JB, Kornacker K: JTK_CYCLE: An efficient nonparametric algorithm for detecting rhythmic components in genome-scale data sets. J Biol Rhythms 2010, 25:372-380.

45. Hughes ME, Grant GR, Paquin C, Qian J, Nitabach MN: Deep sequencing the circadian and diurnal transcriptome of Drosophila brain. Genome Res 2012, 22:1266-1281.

46. Xu K, et al: The circadian clock interacts with metabolic physiology to influence reproductive fitness. Cell Metab 2011, 13:639-654.

47. Keegan KP, Pradhan S, Wang JP, Allada R: Meta-analysis of Drosophila circadian microarray studies identifies a novel set of rhythmically expressed genes. PLoS Comput Biol 2007, 3:e208

48. Wijnen H, Naef F, Boothroyd C, Claridge-Chang A, Young MW: Control of daily transcript oscillations in Drosophila by light and the circadian clock. PLoS Genet 2006, 2:e39.

49. Claridge-Chang A, et al: Circadian regulation of gene expression systems in the Drosophila head. Neuron 2001, 32:657-671.

50. Michael TP, et al: Network discovery pipeline elucidates conserved time-of-day-specific cis-regulatory modules. PLoS Genet 2008, 4:e14.

51. Lin $Y$, et al: Influence of the period-dependent circadian clock on diurnal, circadian, and aperiodic gene expression in Drosophila melanogaster. Proc Natl Acad Sci USA 2002, 99:9562-9567.

52. Harbach RE: The Culicidae (Diptera): A review of taxonomy, classification and phylogeny. Zootaxa 2007, 1668:591-638.

53. Clements AN: The biology of mosquitoes. Oxon: CABI Publ; 1999.

54. Ranson $\mathrm{H}$, et al: Pyrethroid resistance in African anopheline mosquitoes: What are the implications for malaria control? Trends Parasitol 2011, 27:91-98.

55. The malERA Consultative Group on Drugs: A research agenda for malaria eradication: Drugs. PLoS Med 2011, 8:e1000402.

56. Alonso $\mathrm{PL}$, et al: $\mathrm{A}$ research agenda to underpin malaria eradication. PLoS Med 2011, 8:e1000406.

57. Miller BH, et al: Circadian and CLOCK-controlled regulation of the mouse transcriptome and cell proliferation. Proc Natl Acad Sci USA 2007, 104:3342-3347.

58. Rund SSC, Duffield GE: Bioclock, Database of circadian gene expression. Developed by the Duffield laboratory. Notre Dame, IN: University of Notre Dame; 2011. http://nd.edu/ bioclock.

59. Moiroux N, et al: Changes in Anopheles funestus biting behavior following universal coverage of long-lasting insecticidal nets in Benin. J Infect Dis 2012, 206:1622-1629.

60. Ortelli F, Rossiter LC, Vontas J, Ranson H, Hemingway J: Heterologous expression of four glutathione transferase genes genetically linked to a major insecticide-resistance locus from the malaria vector Anopheles gambiae. Biochem J 2003, 373:957-963.

61. Ueda HR, et al: Genome-wide transcriptional orchestration of circadian rhythms in Drosophila. J Biol Chem 2002, 277:14048-14052.

62. Lima-Camara TN, et al: Dengue infection increases the locomotor activity of Aedes aegypti females. PLoS One 2011, 6:e17690.

63. Varghese J, Lim SF, Cohen SM: Drosophila miR-14 regulates insulin production and metabolism through its target, sugarbabe. Genes Dev 2010, 24:2748-2753.

64. Puig O, Marr MT, Ruhf ML, Tjian R: Control of cell number by Drosophila FOXO: downstream and feedback regulation of the insulin receptor pathway. Genes Dev 2003, 17:2006-2020.

65. Djouaka RF, et al: Expression of the cytochrome P450s, CYP6P3 and CYP6M2 are significantly elevated in multiple pyrethroid resistant populations of Anopheles gambiae s.s. from Southern Benin and Nigeria. BMC Genomics 2008, 9:538.

66. Seay DJ, Thummel CS: The circadian clock, light, and cryptochrome regulate feeding and metabolism in Drosophila. J Biol Rhythms 2011, 26:497-506.

67. Kawada H, Takemura SY, Arikawa K, Takagi M: Comparative study on nocturnal behavior of Aedes aegypti and Aedes albopictus. J Med Entomol 2005, 42:312-318.

68. Dunlap JC, et al: A circadian clock in Neurospora: How genes and proteins cooperate to produce a sustained, entrainable, and compensated biological oscillator with a period of about a day. Cold Spring Harb Symp Quant Biol 2007, 72:57-68.

69. Albers HE, Liou SY, Ferris CF, Stopa EG, Zoeller RT: Neurochemistry of circadian timing. In Suprachiasmatic nucleus: The mind's clock. Edited by Klein DC, Moore RY, Reppert SM. New York: Oxford University Press; 1991:263-288.

70. Inouye SIT: Circadian rhythms of neuropeptides in the suprachiasmatic nucleus. Progress in Brain Reserach 1996, 111:75-90.
71. Chen $\mathrm{CH}$, Ringelberg CS, Gross RH, Dunlap JC, Loros JJ: Genome-wide analysis of light-inducible responses reveals hierarchical light signalling in Neurospora. EMBO J 2009, 28:1029-1042.

72. Hastings JW: Biochemical aspects of rhythms - phase shifting by chemicals. Cold Spring Harb Symp Quant Biol 1960, 25:131-143.

73. Biessmann H, Nguyen QK, Le D, Walter MF: Microarray-based survey of a subset of putative olfactory genes in the mosquito Anopheles gambiae. Insect Mol Biol 2005, 14:575-589.

74. Li ZX, Pickett JA, Field LM, Zhou JJ: Identification and expression of odorant-binding proteins of the malaria-carrying mosquitoes Anopheles gambiae and Anopheles arabiensis. Arch Insect Biochem Physiol 2005 58:175-189.

75. Pelosi P, Calvello M, Ban L: Diversity of odorant-binding proteins and chemosensory proteins in insects. Chem Senses 2005, 30(Suppl 1):i291-i292.

76. Nichols Z, Vogt RG: The SNMP/CD36 gene family in Diptera, Hymenoptera and Coleoptera: Drosophila melanogaster, D. pseudoobscura, Anopheles gambiae, Aedes aegypti, Apis mellifera, and Tribolium castaneum. Insect Biochem Mol Biol 2008, 38:398-415.

77. Enayati A, Ranson H, Hemingway J: Insect glutathione transferases and insecticide resistance. Insect Mol Biol 2005, 14:3-8.

78. Pace KE, Baum LG: Insect galectins: Roles in immunity and development. Glycoconj J 2002, 19:607-614.

79. Edery I, Zwiebel LJ, Dembinska ME, Rosbash M: Temporal phosphorylation of the Drosophila period protein. Proc Natl Acad Sci USA 1994, 91:2260-2264.

80. Hardin PE, Hall JC, Rosbash M: Feedback of the Drosophila period gene product on circadian cycling of its messenger RNA levels. Nature 1990 343:536-540.

81. Sehgal A, et al: Rhythmic expression of timeless: $A$ basis for promoting circadian cycles in period gene autoregulation. Science 1995, 270:808-810.

82. Rosbash M, et al: Transcriptional feedback and definition of the circadian pacemaker in Drosophila and animals. Cold Spring Harb Symp Quant Biol 2007, 72:75-84

83. Zheng $X$, Sehgal A: Probing the relative importance of molecular oscillations in the circadian clock. Genetics 2008, 178:1147-1155.

84. Yang Z, Sehgal A: Role of molecular oscillations in generating behavioral rhythms in Drosophila. Neuron 2001, 29:453-467.

85. Reppert SM, Weaver DR: Molecular analysis of mammalian circadian rhythms. Annu Rev Physiol 2001, 63:647-676.

86. Chen $\mathrm{CH}$, Dunlap JC, Loros JJ: Neurospora illuminates fungal photoreception. Fungal Genet Biol 2010, 47:922-929.

87. Ueda HR, et al: System-level identification of transcriptional circuits underlying mammalian circadian clocks. Nat Genet 2005, 37:187-192

88. Kako K, Ishida N: The role of transcription factors in circadian gene expression. Neurosci Res 1998, 31:257-264.

89. Matsumoto A, et al: A functional genomics strategy reveals clockwork orange as a transcriptional regulator in the Drosophila circadian clock. Genes Dev 2007, 21:1687-1700.

90. Shotkoski F, Morris AC, James AA, Ffrench-Constan RH: Functional analysis of a mosquito $\gamma$-aminobutyric acid receptor gene promoter. Gene 1996, 168:127-133.

91. McDonald MJ, Rosbash M, Emery P: Wild-type circadian rhythmicity is dependent on closely spaced E Boxes in the Drosophila timeless promoter. Mol Cell Biol 2001, 21:1207-1217.

92. So W, et al: Takeout, a novel Drosophila gene under circadian clock transcriptional regulation. Mol Cell Biol 2000, 20:6935-6944.

93. Montminy MR, Sevarino KA, Wagner JA, Mandel G, Goodman RH: Identification of a cyclic-AMP-responsive element within the rat somatostatin gene. Proc Natl Acad Sci USA 1986, 83:6682-6686.

94. Lin SC, Lin MH, Horváth P, Reddy KL, Storti RV: PDP1, a novel Drosophila PAR domain bZIP transcription factor expressed in developing mesoderm, endoderm and ectoderm, is a transcriptional regulator of somatic muscle genes. Development 1997, 124:4685-4696.

95. Cyran SA, et al: vrille, Pdp1, and dClock form a second feedback loop in the Drosophila circadian clock. Cell 2003, 112:329-341.

96. Ribbands CR: Moonlight and house-haunting habits of female anophelines in West Africa. Bull Entomol Res 1946, 36:395-417.

97. Charlwood JD, et al: Mating does not affect the biting behaviour of Anopheles gambiae from the islands of São Tomé and Principe, West Africa. Ann Trop Med Parasitol 2003, 97:751-756.

98. Molineaux L: The Garki project: Research on the epidemiology and control of malaria in the Sudan savanna of West Africa. World Health Organization; 1980. 
99. Mathenge EM, et al: Effect of permethrin-impregnated nets on exiting behavior, blood feeding success, and time of feeding of malaria mosquitoes (Diptera: Culicidae) in western Kenya. J Med Entomol 2001 38:531-536.

100. Haddow AJ, Ssenkubuge Y: The mosquitoes of Bwamba County, Uganda IX. Further studies on the biting behaviour of an outdoor population of the Anopheles gambiae Giles complex. Bull Entomol Res 1973, 62:407-414.

101. Taylor B, Jones MDR: The circadian rhythm of flight activity in the mosquito Aedes aegypti (L.): The phase-setting effects of light-on and light-off. J Exp Biol 1969, 51:59-70.

102. Brown MR, et al: Endogenous regulation of mosquito host-seeking behavior by a neuropeptide. J Insect Physio/ 1994, 40:399-406.

103. Huang DW, Sherman BT, Lempicki RA: Systematic and integrative analysis of large gene lists using DAVID bioinformatics resources. Nat Protoc 2009, 4:44-57.

104. Dennis G, et al: DAVID: Database for annotation, visualization, and integrated discovery. Genome Biol 2003, 4:R60.

105. Dardente $H$, et al: Daily and circadian expression of neuropeptides in the suprachiasmatic nuclei of nocturnal and diurnal rodents. Mol Brain Res 2004, 124:143-151.

106. Nunez AA, Bult $A$, McElhinny $T L$, Smale L: Daily rhythms of Fos expression in hypothalamic targets of the suprachiasmatic nucleus in diurnal and nocturnal rodents. J Biol Rhythms 1999, 14:300-306.

107. Martínez GS, Smale L, Nunez AA: Diurnal and nocturnal rodents show rhythms in orexinergic neurons. Brain Res 2002, 955:1-7.

108. Vosko AM, Hagenauer MH, Hummer DL, Lee TM: Period gene expression in the diurnal degu (Octodon degus) differs from the nocturnal laboratory rat (Rattus norvegicus). Am J Physiol Regul Integr Comp Physiol 2009, 296:R353-R361.

109. Patrick ML, Aimanova K, Sanders HR, Gill SS: P-type $\mathrm{Na}^{+} / \mathrm{K}^{+}$-ATPase and V-type $\mathrm{H}^{+}$-ATPase expression patterns in the osmoregulatory organs of larval and adult mosquito Aedes aegypti. J Exp Biol 2006, 209:4638-4651.

110. Calvo E, Dao A, Pham VM, Ribeiro JMC: An insight into the sialome of Anopheles funestus reveals an emerging pattern in anopheline salivary protein families. Insect Biochem Mol Biol 2007, 37:164-175.

111. Klein U, Zimmermann B: The vacuolar-type ATPase from insect plasma membrane: Immunocytochemical localization in insect sensilla. Cell Tissue Res 1991, 266:265-273.

112. Sessions $\mathrm{OM}$, et al: Discovery of insect and human dengue virus host factors. Nature 2009, 458:1047-1050.

113. Nawa M: Effects of bafilomycin $A 1$ on Japanese encephalitis virus in C6/36 mosquito cells. Arch Virol 1998, 143:1555-1568.

114. Cociancich SO, Park SS, Fidock DA, Shahabuddin M: Vesicular ATPaseoverexpressing cells determine the distribution of malaria parasite oocysts on the midguts of mosquitoes. J Biol Chem 1999, 274:12650-12655.

115. Beyenbach KW, Wieczorek $\mathrm{H}$ : The V-type $\mathrm{H}^{+}$ATPase: Molecular structure and function, physiological roles and regulation. J Exp Biol 2006, 209:577-589.

116. Moriyama Y, Maeda M, Futai M: The role of V-ATPase in neuronal and endocrine systems. J Exp Biol 1992, 172:171-178.

117. Hiesinger PR, et al: The v-ATPase $V_{0}$ subunit a1 is required for a late step in synaptic vesicle exocytosis in Drosophila. Cell 2005, 121:607-620.

118. Williamson WR, Wang D, Haberman AS, Hiesinger PR: A dual function of $\mathrm{V}_{0}$-ATPase a1 provides an endolysosomal degradation mechanism in Drosophila melanogaster photoreceptors. J Cell Biol 2010, 189:885-899.

119. Pyza E, Borycz J, Giebultowicz JM, Meinertzhagen IA: Involvement of V-ATPase in the regulation of cell size in the fly's visual system. J Insect Physiol 2004, 50:985-994.

120. Zuker CS: The biology of vision of Drosophila. Proc Natl Acad Sci USA 1996, 93:571-576

121. Katz B, Minke B: Drosophila photoreceptors and signaling mechanisms. Front Cell Neurosci 2009, 3:2

122. Han J, et al: The fly CAMTA transcription factor potentiates deactivation of rhodopsin, a G protein-coupled light receptor. Cell 2006, 127:847-858

123. Colley NJ, Baker EK, Stamnes MA, Zuker CS: The cyclophilin homolog ninaA is required in the secretory pathway. Cell 1991, 67:255-263.

124. Wang T, Wang X, Xie Q, Montell C: The SOCS box protein STOPS is required for phototransduction through its effects on phospholipase $C$. Neuron 2008, 57:56-68.

125. Fleissner G, Fleissner G: Efferent control of visual sensitivity in arthropod eyes: With emphasis on circadian rhythms. Stuttgart, New York: G. Fischer Verlag; 1987.
126. Yau KW, Hardie RC: Phototransduction motifs and variations. Cell 2009, 139:246-264.

127. Zhou JJ, He XL, Pickett JA, Field LM: Identification of odorant-binding proteins of the yellow fever mosquito Aedes aegypti: Genome annotation and comparative analyses. Insect Mol Biol 2008, 17:147-163.

128. Benton R, Sachse S, Michnick SW, Vosshall LB: Atypical membrane topology and heteromeric function of Drosophila odorant receptors in vivo. PLOS Biol 2006, 4:e20.

129. Wise de Valdez MR, et al: Genetic elimination of dengue vector mosquitoes. Proc Natl Acad Sci USA 2011, 108:4772-4775.

130. Meredith JM, et al: Site-specific integration and expression of an anti-malarial gene in transgenic Anopheles gambiae significantly reduces Plasmodium infections. PLOS One 2011, 6:e14587.

131. Matsumoto A, et al: Period gene of Bactrocera cucurbitae (Diptera: Tephritidae) among strains with different mating times and sterile insect technique. Ann Entomol Soc Am 2008, 101:1121-1130.

132. Fuchikawa T, et al: The clock gene cryptochrome of Bactrocera cucurbitae (Diptera: Tephritidae) in strains with different mating times. Heredity 2010, 104:387-392.

133. Mbogo CNM, Baya NM, Ofulla AVO, Githure Jl, Snow RW: The impact of permethrin-impregnated bednets on malaria vectors of the Kenyan coast. Med Vet Entomol 1996, 10:251-259.

134. Lawson D, et al: VectorBase: A data resource for invertebrate vector genomics. Nucleic Acids Res 2009, 37:D583-D587.

135. Xi Z, Ramirez JL, Dimopoulos G: The Aedes aegypti toll pathway controls dengue virus infection. PLoS Pathog 2008, 4:e1000098.

136. Cooley JW, Tukey JW: An algorithm for the machine calculation of complex Fourier series. Math Computation 1965, 19:297-301.

137. Cook C, Bernfeld M: CH 2. Matched Filter, Radar signals: An introduction to theory and application. Boston: Artech House; 1993

138. Hunter S, et al: InterPro: The integrative protein signature database. Nucleic Acids Res 2009, 37:D211-D215.

139. Eisen MB, Spellman PT, Brown PO, Botstein D: Cluster analysis and display of genome-wide expression patterns. Proc Natl Acad Sci USA 1998, 95:14863-14868.

140. Saldanha AJ: Java Treeview-extensible visualization of microarray data. Bioinformatics 2004, 20:3246-3248.

141. Dana AN, et al: Gene expression patterns associated with blood-feeding in the malaria mosquito Anopheles gambiae. BMC Genomics 2005, 6:5.

142. Müller $P$, Donnelly MJ, Ranson H: Transcription profiling of a recently colonised pyrethroid resistant Anopheles gambiae strain from Ghana. BMC Genomics 2007, 8:36.

143. Biessmann $\mathrm{H}$, et al: The Anopheles gambiae Odorant Binding Protein 1 (AgamOBP1) mediates indole recognition in the antennae of female mosquitoes. PLoS One 2010, 5:e9471.

doi:10.1186/1471-2164-14-218

Cite this article as: Rund et al:: Extensive circadian and light regulation of the transcriptome in the malaria mosquito Anopheles gambiae. BMC Genomics 2013 14:218.

\section{Submit your next manuscript to BioMed Central and take full advantage of:}

- Convenient online submission

- Thorough peer review

- No space constraints or color figure charges

- Immediate publication on acceptance

- Inclusion in PubMed, CAS, Scopus and Google Scholar

- Research which is freely available for redistribution 